\title{
A typology of fisheries management tools: using experience to catalyse greater success
}

\author{
Elizabeth R Selig ${ }^{1, a, *}$, Kristin M Kleisner ${ }^{2, b}, *$, Oren Ahoobim ${ }^{3}$, Freddy Arocha ${ }^{4}$, Annabelle Cruz-Trinidad ${ }^{5}$, \\ Rod Fujita ${ }^{6}$, Mafaniso Hara ${ }^{7}$, Laure Katz ${ }^{1}$, Patrick McConney ${ }^{8}$, Blake D Ratner ${ }^{9}$, Lina M Saavedra-Díaz ${ }^{10}$, \\ Anne-Maree Schwarz ${ }^{11}$, Djiga Thiao ${ }^{12}$, Elin Torell ${ }^{13}$, Sebastian Troëng ${ }^{1,14}$ E Sebastian Villasante ${ }^{15,16}$
}

${ }^{1}$ Betty and Gordon Moore Center for Science and Oceans, Conservation International, 2011 Crystal Drive Suite 500, Arlington, VA 22202, USA; ${ }^{2}$ Sea Around Us Project, University of British Columbia, 2202 Main Mall, Vancouver, BC V6T 1Z4, Canada; ${ }^{3}$ Dalberg Global Development Advisors, 99 Madison Avenue, New York, NY 10016, USA; ${ }^{4}$ Department of Fishery Biology, Instituto Oceanográfico de Venezuela, Universidad de Oriente, Cumaná, Venezuela; ${ }^{5}$ FishBase Information and Research Group, Inc. (FIN), Los Baños, 4031 Laguna, Philippines; ${ }^{6}$ Environmental Defense Fund, 123 Mission St, 28th Floor, San Francisco, CA 94105, USA; ${ }^{7}$ Institute for Poverty, Land and Agrarian Studies (PLAAS), University of the Western Cape, Private Bag X17, Bellville 7535, South Africa; ${ }^{8}$ Centre for Resource Management and Environmental Studies, The University of the West Indies, Cave Hill, Barbados; ${ }^{9}$ WorldFish, Jalan Batu Maung, Batu Maung, 11960 Bayan Lepas, Penang, Malaysia; ${ }^{10}$ Department of Biology, Universidad del Magdalena, P.O. Box 2-1-21630, Carrera 32 No. 22 - 08, Santa Marta - Magdalena, Colombia; ${ }^{11}$ WorldFish, P.O. Box 438, Honiara, Solomon Islands; ${ }^{12}$ Centre de Recherches Océanographiques de Dakar-Thiaroye, BP 2241 Dakar, Senegal; ${ }^{13}$ Coastal Resources Center, University of Rhode Island, 220 South Ferry Road, Narragansett, RI 02882, USA; ${ }^{14}$ Department of Biology, Lund University, Lund, Sweden; ${ }^{15}$ Faculty of Political and Social Sciences, University of Santiago de Compostela, Av. Angel Echevarry s/n, 15782 A Coruña, Spain; ${ }^{16}$ Natural Capital Project, Stanford University, 371 Serra Mall, Stanford, CA 94305, USA; ${ }^{a}$ Present address: Norwegian Institute for Water Research, Gaustadalléen 21, 0349 Oslo, Norway; ${ }^{b}$ Present address: Environmental Defense Fund, 18 Tremont St, Ste 850, Boston, MA 02108, USA

\begin{abstract}
Fisheries provide nutrition and livelihoods for coastal populations, but many fisheries are fully or over-exploited and we lack an approach for analysing which factors affect management tool performance. We conducted a literature review of 390 studies to assess how fisheries characteristics affected management tool performance across both small-scale and large-scale fisheries. We defined success as increased or maintained abundance or biomass, reductions in fishing mortality or improvements in population status. Because the literature only covered a narrow set of biological factors, we also conducted an expert elicitation to create a typology of broader fishery characteristics, enabling conditions and design considerations that affect performance. The literature suggested that the most commonly used management tool in a region was often the most successful, although the scale of success varied. Management tools were more often deemed successful when used in combination, particularly pairings of tools that controlled fishing mortality or effort with spatial management. Examples of successful combinations were the use of catch limits with quotas and limited entry, and marine protected areas with effort restrictions. The most common factors associated with inadequate biological performance were 'structural' issues, including poor design or implementation. The expert-derived typologies revealed strong local leadership, high community involvement and governance
\end{abstract}

Correspondence: Elizabeth R Selig, Norwegian Institute for Water Research, Gaustadalléen 21 , 0349, Oslo, Norway Tel.: (+47) 221851 00 Fax: (+47) 221852 00 E-mail: elizabeth selig@niva.no

*Authors contributed equally to this work. Received 26 Feb 2016

Accepted 8 Sep 2016 
capacity as common factors of success across management tool categories (i.e. input, output and technical measures), but the degree of importance varied. Our results are designed to inform selection of appropriate management tools based on empirical data and experience to increase the likelihood of successful fisheries management.

Keywords Fisheries management, input controls, management effectiveness, output controls, small-scale fisheries, spatial management

$\begin{array}{ll}\text { Introduction } & \mathbf{2} \\ \text { Methods } & \mathbf{5} \\ \text { Literature review } & 5 \\ \text { Classification trees } & 7 \\ \text { Classification tree validation } & 8 \\ \text { Expert-derived typology } & 8 \\ \text { Results } & \mathbf{9} \\ \text { Literature review findings } & 9 \\ \text { Classification trees } & 10 \\ \text { Expert-derived typology } & 11 \\ \text { Discussion } & \mathbf{1 5} \\ \text { Patterns within management tool categories } & 22 \\ \quad \text { Input controls } & 22 \\ \quad \text { Output controls } & 22 \\ \quad \text { Technical measures } & 23 \\ \text { Integrating 'lessons learned' into the process of determining a fisheries } & 23 \\ \text { management approach } & 23 \\ \text { Acknowledgements } & 25 \\ \text { Supporting Information } & \mathbf{2 5}\end{array}$

\section{Introduction}

Wild-capture fisheries are a critical source of food and employment. They provide dietary protein and micronutrients for impoverished communities that may not have access to alternative sources of nutrition (FAO, 2014) and contribute to livelihoods and income for an estimated 260 million people worldwide (Teh and Sumaila 2013). In many parts of the developing world, these livelihood and nutritional benefits from marine resources are derived locally, from communities that fish in coastal and inland waters near their homes (FAO, 2014). Given the importance of wildcapture fisheries as a sustainable resource that provides essential nutrition and livelihoods, effective management is necessary to ensure productivity and maintain these benefits.

Several analyses have examined the factors that contribute to the successful implementation of a particular management tool (e.g. catch shares; Essington et al. 2012) or management approach (e.g. co-management; Gutierrez et al. 2011; Cinner et al. 2012), but none have explored the factors that impact the performance of a suite of management tools. Many studies suggest that management tools may be more or less successful depending on the context, that is governance structure, which determines the rules of access and whether fisheries are communal property or governed by individual or collective rights-based management (Stefansson and Rosenberg 2005; Mahon et al. 2008; Gutierrez et al. 2011; Cinner et al. 2012; Essington et al. 2012; Yamazaki et al. 2012; Maggs et al. 2013; Wielgus et al. 2014).

Defining 'success' in fisheries management can be challenging given different management objectives. Historically, a primary goal of fisheries management is the control of fishing mortality such that stocks can maintain themselves at levels 
capable of providing desired yields on a continuing basis (Hilborn and Walters 1992). However, the broader reasons for maintaining sufficient yields are often context-specific and can include increasing or maintaining profitability for private operators and the fishing industry, increasing national export revenue, improving local food security (Béné et al. 2006) or maintaining cultural identity (Urquhart and Acott 2014). The diagnosis and design of fisheries management options must recognize and reflect the particular mix and priority of stakeholder objectives in a given location (Andrew et al. 2007; Poon et al. 2013). This may be especially true in small-scale fisheries because those involved in the fisheries have close ties to the community or in co-managed fisheries where governance may occur at multiple levels including the community scale (Weeratunge et al. 2013). Management tools provide a means to help achieve these objectives across both small- and large-scale fisheries.

Here, we considered three general categories of fishery management tools: input controls, output controls and technical measures (Table 1; Sutinen and Soboil 2003). In general, input controls limit the amount of fishing effort as a way to control the amount of fish caught, while output controls are direct limits on the amount of fish harvested. For the purposes of this paper, technical measures are controls on where and when fishermen may fish and the size of fish they may harvest. Because of the scale (spatial, temporal, jurisdictional, etc.) at which different management tools are implemented and the type of control they exert, they may have direct or indirect effects on achieving fisheries management objectives. Additionally, the effectiveness of all management interventions can be limited to varying degrees by the scale at which important biological, ecological, socioeconomic and governance processes apply to a particular species or suite of species on which the fishery is operating. For example, a catch limit could result in a desired level of fishing mortality across an entire fishery, whereas an MPA (or network of MPAs) typically reduces fishing mortality in a relatively small portion of a stock's range, potentially resulting in a smaller impact on total stock-level biomass. While acknowledging these difficulties, we attempted to generally categorize direct and indirect effects of different management tools (Table 2), noting that the borders between these categories are not rigid and assignments may vary depending on context and location.

We took two approaches to assessing the performance of different management tools. The first approach was a systematic literature review to determine how fishery characteristics, including geography, scale of fisheries management and type of stock, affect the performance of different fisheries management tools. In the literature review, we considered both small-scale and largescale marine fisheries because both types can provide useful information about factors that affect performance and we wanted to examine all management tools, some of which were more or less common in small- or large-scale fisheries. We evaluated management tool performance using a relatively narrow definition of success. We defined 'success' as an improvement in or maintenance of the abundance or biomass of the species, a reduction of fishing mortality or an improvement in the status of the population to sustainable levels.

There are many other metrics of performance across social, cultural and economic axes (Béné et al. 2006, 2010; Allison et al. 2012; Weeratunge et al. 2013; Hicks et al. 2014). Our definition of success focused on biological improvement

Table 1 Input, output and technical management tools considered in this study based on Sutinen and Soboil (2003).

\begin{tabular}{llc}
\hline Input & Output & Technical measures \\
\hline Limited entry (e.g. licensing) & Total allowable catch & Marine protected areas \\
$\begin{array}{c}\text { Time restriction } \\
\text { (i.e. days at sea, seasonal restrictions) }\end{array}$ & $\begin{array}{l}\text { Individual transferrable quotas, } \\
\text { catch shares }{ }^{1}\end{array}$ & Time-area closures \\
Gear restrictions & (i.e. seasonal area & closures, temporary closures) \\
& Territorial user rights fisheries & Size limits
\end{tabular}

${ }^{1}$ Catch shares are defined here as species-based shares. TURFs are defined as area-based shares. 
Table 2 Goals associated with different management tools. A direct effect is defined as a tool that is designed to produce a desired goal (e.g. limiting the amount of fishing that can be taken will theoretically result in lower fishing mortality). An indirect effect provides a control mechanism that operates on a different facet of the system, but can achieve the desired goal (e.g. an ITQ can protect habitat if the deployment of habitat damaging gear is limited).

\begin{tabular}{|c|c|c|c|c|}
\hline \multirow[b]{2}{*}{ Management tools } & \multicolumn{4}{|l|}{ Goals } \\
\hline & Control fishing mortality & Restore age-structure & $\begin{array}{l}\text { Protect marine } \\
\text { habitat }\end{array}$ & $\begin{array}{l}\text { Protect subpopulation } \\
\text { structure to preserve } \\
\text { genetic diversity }\end{array}$ \\
\hline Limited entry (e.g. licensing) & 1 & & & \\
\hline Gear restrictions & 1 & $\mathrm{D}$ & $\mathrm{D}$ & \\
\hline Time restrictions & 1 & & & $D^{2}$ \\
\hline Total allowable catch & $\mathrm{D}$ & 1 & & \\
\hline Quotas (e.g. catch shares and ITQs) & $\mathrm{D}$ & 1 & 1 & \\
\hline Territorial user rights fisheries & $\mathrm{I}^{1}$ & $\mathrm{I}^{1}$ & $\mathrm{D}$ & $\mathrm{D}$ \\
\hline Marine protected areas & $I^{1}$ & $1^{1}$ & $\mathrm{D}$ & $\mathrm{D}$ \\
\hline Time-area closures & $1^{1}$ & $1^{1}$ & $\mathrm{D}$ & 1 \\
\hline Size limits & I & $\mathrm{D}$ & & \\
\hline
\end{tabular}

ITQs, individual transferrable quotas.

${ }^{1}$ Indicates that a tool could be used to achieve that goal if it is large enough.

${ }^{2}$ Indicates that the tool could be effective if implemented during spawning season.

because biological metrics were the only indicators consistently reported across studies. The lack of consistent reporting outside of biological variables is a known problem in evaluating fisheries management, particularly for measuring social and contextual factors (Wamukota et al. 2012; Marchal et al. 2016). Our efforts to quantitatively assess performance were constrained by what was available in the literature. Therefore, we did not evaluate whether management resulted in any trade-offs or increases in seafood availability, improvement in livelihoods or other socioeconomic factors because this information was generally not consistently reported in similar metrics or was simply not available from the majority of studies we reviewed.

We also used this particular definition of success as a means of standardizing responses because success can be context-specific and management tool-related. For example, a stringent catch limit or large MPA that almost eliminates fishing activity might be viewed as 'successful' from a conservation perspective, but may be considered by others to be socially or economically inadequate (Anderson et al. 2015). If a management tool did not achieve the objectives of 'biological success' as we defined them, it was considered 'inadequate'.
Although we could not find consistent metrics other than those related to 'biological success', the literature review did enable us to understand geographic patterns in the application of management tools, quantify current rates of success and identify which biological fisheries characteristics may be related to performance.

To capture other dimensions of 'success' across multiple fisheries scales and because the set of fishery characteristics that were consistently discussed in the literature was limited to functional aspects of the fishery and biological metrics (e.g. fisheries region, mobility of target species, single- vs. multispecies fishery, gear type, habitat type, fisheries management approach and scale of management), we solicited the input of experts to create performance typologies. These complementary expert elicitation typologies included not only a broader set of fisheries characteristics than what could be directly evaluated from the literature, but also the enabling conditions and design considerations important to the successful implementation of management tools. The expert elicitation focused on how various factors may affect conditions in small-scale fisheries in particular, but results are applicable across both large- and small-scale fisheries. 
We designed this study only to help managers determine which management tools may have a greater probability of biological success given the particular characteristics of their fishery. The available data were not sufficient to determine the 'best' management tool for a given context particularly given the lack of available social, economic or cultural metrics from the literature review, nor do we feel it would be appropriate to aim for this level of determinism. Nonetheless, our results can be used to facilitate a design approach that considers the local context and management goals to select the appropriate management tool based on empirical information from the literature and expert experience on the factors that affect performance over time in a given context.

\section{Methods}

\section{Literature review}

The goal of the literature review was to find examples of the efficacy of various management tools and strategies from English-language primary and grey literature with a focus on small-scale fisheries in developing countries (Fig. 1), but including industrial fisheries in developed countries as well. As mentioned above, we characterized the application of a management tool as a 'success' if there was biological improvement, defined as an increase in the abundance, biomass or evidence of stock recovery, in the population or group of species being managed as determined by the author of the specific publication or case-study. If it was unclear whether or not there was an improvement in the abundance of the stock or group of species, we did not include the study in our review. A management tool was defined as 'inadequate' if there was a decrease in abundance, biomass or stock status below a level that was deemed sustainable. In other words, some reduction in biomass might be acceptable with some management tools such as catch, size or effort limits if the goal is to reduce the stock to a reference level such as the biomass level at maximum sustainable yield $\left(\mathrm{B}_{\mathrm{MSY}}\right)$. However, reductions beyond such a reference limit would indicate that the management tool was performing inadequately. Studies that reflected partial improvement, that is goals were not completely met, but there were some positive biological changes noted, were also classified as 'successful' in the analysis because the trajectory reflected an improvement.

Where possible, we also attempted to classify the reasons for inadequacies, noting whether management tools performed poorly due to 'structural' shortcomings, 'external pressures' or 'compliance'related issues. Structural shortcomings were defined as problems associated with planning and implementation including those due to design, for example the choice of the wrong management tool for the situation or a process, or inadequate

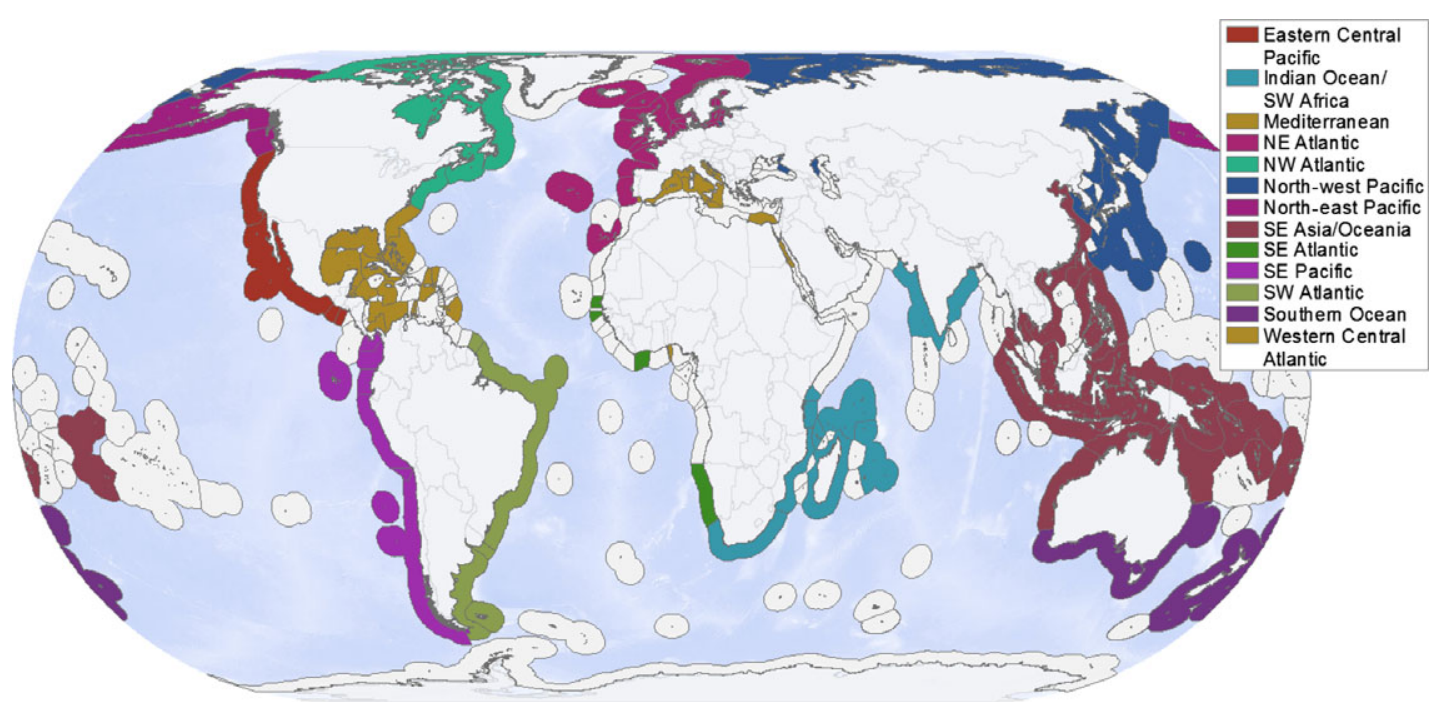

Figure 1 The 13 regions analysed, grouped by EEZ areas. These regions do not pertain to actual political or ecological boundaries, but were based on the major regional groupings found in the literature review. 
consultation and dialogue with stakeholders during the planning stages. External pressures were defined as pressures from outside the fishery such as climate change or external fishing pressures, for example foreign commercial fishing. Finally, compliance issues were defined as those that arise when rules and regulations are not respected or enforced. The reasons that tools did not performed adequately can highlight the importance of considering the social and governance context in which tools are implemented.

Although there are many important ecosystemlevel management approaches and indicators, we limited our literature review to management tools (Table 1) for species or groups of species that are caught for human consumption and are directly targeted (i.e. not by-catch) to maintain analytical tractability. We used a structured search on key terms for the major categories of management tools under the input, output and technical measure categories (Sutinen and Soboil 2003; Table S1) for studies from 1990 to 2014. In order to define a unique record, we identified individual stocks when possible by region, which were identified by fishing country and major oceanic area (e.g. North-west Atlantic) or body of water. When more than one study describing the same management strategy or tool in the same area was available, we selected the most recent study (typically in the 2000s) and included information from the older study within the record when it provided key supporting information on the outcome of the management tool. When two individual studies presented information on the use of different management tools for the same stock in the same region, we created two unique records. We did not include studies that dealt only with theoretical applications of management tools and restricted our analysis to those citing actual improvements to the fishery for a given species or set of species that are fished for direct consumption, by commercial fisheries, that is not recreational or trophy fishing.

The assignment of particular management tools to the categories of input, output and technical measures can be considered somewhat subjective. Input controls are generally considered to be limits on the amount or type of effort fishers put into their fishing activities, indirectly controlling the amount of fish caught (Kura et al. 2004). Here, we considered limited entry (e.g. licensing) and time and gear restrictions to be input controls.
Output controls directly limit the amount of fish that can be harvested. Output controls consist of overall limits on the catch of a given stock (i.e. total allowable catch [TAC]), which can then be either fished on a competitive basis or divided up between the participants in the fishery so that each fisher or group has a defined share of the catch (Fujita et al. 1996; Poon et al. 2013). We analysed species-based catch shares including individual transferable quotas (ITQs), as a group, and territorial user rights fisheries (TURFs), separately. 'Catch share' is a general term for several fishery management strategies that allocate a specific portion of the total allowable fishery catch to individuals, cooperatives, communities or other entities. Each recipient of a catch share is directly accountable to stop fishing, when its exclusive allocation is reached. Here we defined catch shares and ITQs as a single management tool, which we refer to as 'quotas', even though transferability can affect catch share performance (Criddle and Strong 2013), because the papers were not always clear whether the quotas were transferrable or not. In this study, technical measures were considered to be marine protected areas (MPAs), time-area closures and limits on the size of fish that can be caught (Sutinen and Soboil 2003). MPAs were defined here as a long-term restriction on the types of activities that can occur within spatial boundaries. MPAs can include both no-take reserves (where all fishing is restricted) and multiuse areas where only particular gears or certain types or times of fishing are restricted.

In assessing each example, we evaluated whether the given management tool or tools used in each case-study was biologically successful. Additional information was gathered in order to try to characterize factors that might relate to this success (Table 3). These variables were limited to what was consistently reported in the literature.

As a measure of robustness, we asked an independent researcher to review $10 \%$ of the 390 studies and conduct the same classification we did to ensure that our classification was repeatable. We used a random number generator to select the studies for which the researcher repeated the classification. We then compared how much congruence there was between the classification of 'success' vs. 'inadequate'. We also compared the congruence between the independent researcher's classification and our initial classification for all other variables we used in the analysis, including 
Table 3 Fishery characteristics identified in the literature review. Note that coastal population and Human Development Index were external globally available variables that were assigned based on the country listed in each study.

\begin{tabular}{|c|c|}
\hline Fishery characteristic & Category \\
\hline Body of water or ocean region & $\begin{array}{l}\text { Eastern Central Pacific (ECP), Indian Ocean (IO)/SW Africa, Mediterranean } \\
\text { (Med), North-east Atlantic (NEA), North-east Pacific (NEP), North-west } \\
\text { Atlantic (NWA), North-west Pacific (NWP), South-east Atlantic (SEA), } \\
\text { South-East Asia/Oceania (SEAO), Southwest Atlantic (SWA), South-east } \\
\text { Pacific (SEP), the Southern Ocean (SO) and Western Central Atlantic } \\
\text { (WCA) }\end{array}$ \\
\hline Mobility & Sessile/Low mobility (S) or Mobile (M) \\
\hline Single or multispecies fishery & Single target species or multiple target species \\
\hline Habitat or ecosystem fished & $\begin{array}{l}\text { Benthic }(B) \text {, Demersal }(D) \text {, Pelagic }(P) \text {, Reef }(R) \text {, Benthic/Demersal }(B / D) \text {, } \\
\text { Pelagic/Demersal }(P / D)\end{array}$ \\
\hline Main gear used & $\begin{array}{l}\text { Bottom trawl, Dredge, Hand harvest, Line fishing, Longline, Nets, } \\
\text { Purse seine, Traps }\end{array}$ \\
\hline Transboundary stock & Yes, No \\
\hline Fisheries management approach & $\begin{array}{l}\text { Ecosystem-based, rights-based, yield optimization, customary marine tenure, } \\
\text { conservation approach }\end{array}$ \\
\hline Scale of fisheries management & Community $(\mathrm{C})$, co-management $(\mathrm{Co}-\mathrm{M})$, national $(\mathrm{N})$ \\
\hline Is tool used in combination with other tools? & Yes, No \\
\hline $\begin{array}{l}\text { Coastal population within } 100 \mathrm{~km} \text { of coastline } \\
\text { (CIESIN, 2012) }\end{array}$ & Low ( $<50 \%$ quartile), medium ( $50 \%$ quartile), high ( $75 \%$ quartile) \\
\hline $\begin{array}{l}\text { Classified Human Development Index } \\
\text { (United Nations Development Programme 2011) }\end{array}$ & Low ( $<50 \%$ quartile), medium ( $50 \%$ quartile), high ( $75 \%$ quartile) \\
\hline
\end{tabular}

single vs. multispecies, degree of mobility, the ecosystem within which the tool was implemented, the particular tool or tools that were implemented and the management approach used.

\section{Classification trees}

We used classification trees to determine which fisheries characteristics were most related to the successful implementation of each management tool, individually (De'ath and Fabricius 2000). Classification trees were implemented in the $\mathrm{R}$ package rpart (Therneau et al. 2014). We tested several independent categorical variables gleaned from each study: the mobility of the species, whether the fishery targets a single or multiple species, ecosystem type, fisheries management approach, scale of management and whether or not that management tool was used in combination with other management tools (Table 3). In addition to these variables derived from the literature, we also included globally available indicators such as Human Development Index score (HDI; United Nations Development Programme, 2011), and coastal population density within $100 \mathrm{~km}$ of the shoreline (CIESIN, 2012) (Table 3) because degree of development and population characteristics may impact the resources available for management. Because we examined factors of success that related to a given management tool, unequal sample sizes between tools should not have an effect on results across tools, although smaller sample sizes may reduce confidence in patterns for a particular tool.

Classification trees are particularly useful for the evaluation of multiple decision points when the data are binomial and categorical (Loh 2011). Classification trees are designed for dependent variables that take a finite number of unordered values. One of their key benefits is that they provide a visual interpretation of patterns. However, one of the drawbacks is that prediction error is measured in terms of misclassification cost, a measure that is not as robust as prediction error measured by the squared difference between the observed and predicted values. Prediction error measured in these terms would be attainable from a regression tree, which are used when the dependent variables take continuous or ordered discrete values (Loh 2011). 


\section{Classification tree validation}

To generally validate the classification trees and because regression trees were not appropriate for our data, we ran binomial generalized linear models (GLMs) with link = 'logit' for all tools to confirm the first split in the classification trees. We chose not to use GLMs exclusively because they will not determine broader thresholds where a tool may have greater success (e.g. low HDI vs. medium-high HDI) and because we wanted to evaluate multiple decision boundaries (e.g. a particular tool may work best if both $\mathrm{X}$ and $\mathrm{Y}$ conditions are present), which is a strength of the classification tree approach. However, classification trees are prone to overfitting, a drawback to which GLMs are more robust. GLMs will only determine which factor level within each variable is most significant. We only present trees for tools that had enough observations to be evaluated with the binomial GLMs (i.e. they could not be used for time restrictions, TACs or TURFs) and also pruned the trees to illustrate only the first two splits. Splits beyond the second node of the trees are based on too few observations and may present erroneous patterns. The binomial GLMs were then used to confirm the splits in the trees. The GLMs were conducted on the same categorical variables we used for the classification trees. Because of the inherent statistical differences in the methods, we did not expect to have congruence between the factor levels deemed significant, but instead used the GLM to confirm the general importance of the categorical variable defining the first split in the tree.

\section{Expert-derived typology}

We convened an expert working group of 19 diverse fisheries scientists, economists and resource managers with expertise on fisheries in Senegal, South Africa, the Philippines, the Solomon Islands and broader regional experience with fisheries in the Caribbean, South America, Europe, the USA and South-East Asia to vet the literature review, address potential publication bias and consider a wider variety of potential factors of success by drawing on their experience with a broad range of fisheries. Working group members were asked to identify key fisheries characteristics that might determine whether a particular management tool would be successful. For example, if a species has high mobility, management tools that are spatially fixed may be less effective. For the most part, the expert working group focused on fisheries characteristics that were not consistently reported in the literature.

In addition to fishery characteristics, working group members also identified a series of enabling conditions that may affect performance. Enabling conditions generally encompass a suite of governance, community and capacity-related factors that can vary over space and time and may influence the degree of success of a particular management intervention. Enabling conditions generally could not be extracted from the literature review because there tended to be a separation between papers that assessed management success in biological terms and those that described the socioeconomic context of fishing communities. Very few papers covered both aspects (but see Moreno-Sanchez and Maldonado 2013; Batista et al. 2011) and analysing interactions across separate studies was not tractable. Experts were able to specify links between certain kinds of enabling conditions and potential success of different management tools. For example, nesting catch limits within a governance structure that provides secure harvest privileges (e.g. ITQs or collectively held privileges) can alleviate race-to-fish behaviour, improving compliance with the catch limit and reducing discard rates. Secure harvest privileges are also correlated with the implementation of conservation measures by fishery cooperatives (Ovando et al. 2013).

Experts agreed that in addition to fishery characteristics and enabling conditions, several design considerations for each management tool were also important determinants of success. Many of these factors should be considered during the design phase of any management tool. Some factors may affect the success of the acceptance of the management tool by local communities and the likelihood that they will comply with the regulations. Like enabling conditions, design considerations were not generally available from the literature review, as most studies did not include a discussion of the process used to implement the management tools. Experts ranked each of the fishery characteristics, enabling conditions and design considerations by their degree of importance for the successful implementation of a given management tool to create three typology tables. 


\section{Results}

\section{Literature review findings}

We had 390 studies that had information that could be used to classify the performance of different management tools (See Supporting Information for references of studies used in our analyses). The majority of the studies were post-2000, but a few were from the late 1990s. The studies came from 13 regions (Figs 1 and 2): Eastern Central Pacific (ECP), Indian Ocean (IO)/SE Africa, Mediterranean (Med), North-east Atlantic (NEA), North-east Pacific (NEP), North-west Atlantic (NWA), North-west Pacific (NWP), South-east Atlantic (SEA), South-East Asia/Oceania (SEAO), South-west Atlantic (SWA), South-east Pacific (SEP), the Southern Ocean (SO) and Western Central Atlantic (WCA). Of the 390 studies, 159 dealt specifically with small-scale fisheries (SSF) and 72 dealt with a combination of small-scale and largescale fisheries.

In the literature, the types of management tools that were implemented varied by region, but all regions included input, output and technical measures (Table S2). In most regions, the ratio of input to output and technical measures was relatively even. However, output controls were less common in the Mediterranean and the SE Atlantic
(West African coast), and input controls were less common in the SE Pacific (Pacific coast of South America), where TURFs, quotas and MPAs dominate (Table S2). MPAs were implemented most frequently in the Eastern Central Pacific, the Indian Ocean, the Mediterranean and SE Asia/Oceania according to the literature available. Quotas were also frequently applied (Table S2) and were more successful in the North Atlantic and the Southern Ocean regions (Table 4). Success rates were also affected by whether a management tool was implemented alone or in combination. For example, in the NW Pacific, limited entry and time-area closures were used in combination with other tools in $78 \%$ of the successful cases.

We also identified the most common inadequate' management tool in each region. We found that different tools had varying rates of inadequate performance in different regions. Importantly, we found that structural shortcomings (i.e. problems in the planning or implementation stages) were the most common reasons for poor performance, followed by compliance issues (Fig. 3).

The robustness test of our classification suggests that our methods were repeatable. There was very high congruence $(95 \%)$ in the classification of successful vs. inadequate performance between the independent classification and our classification. For all other factors assessed - identification of

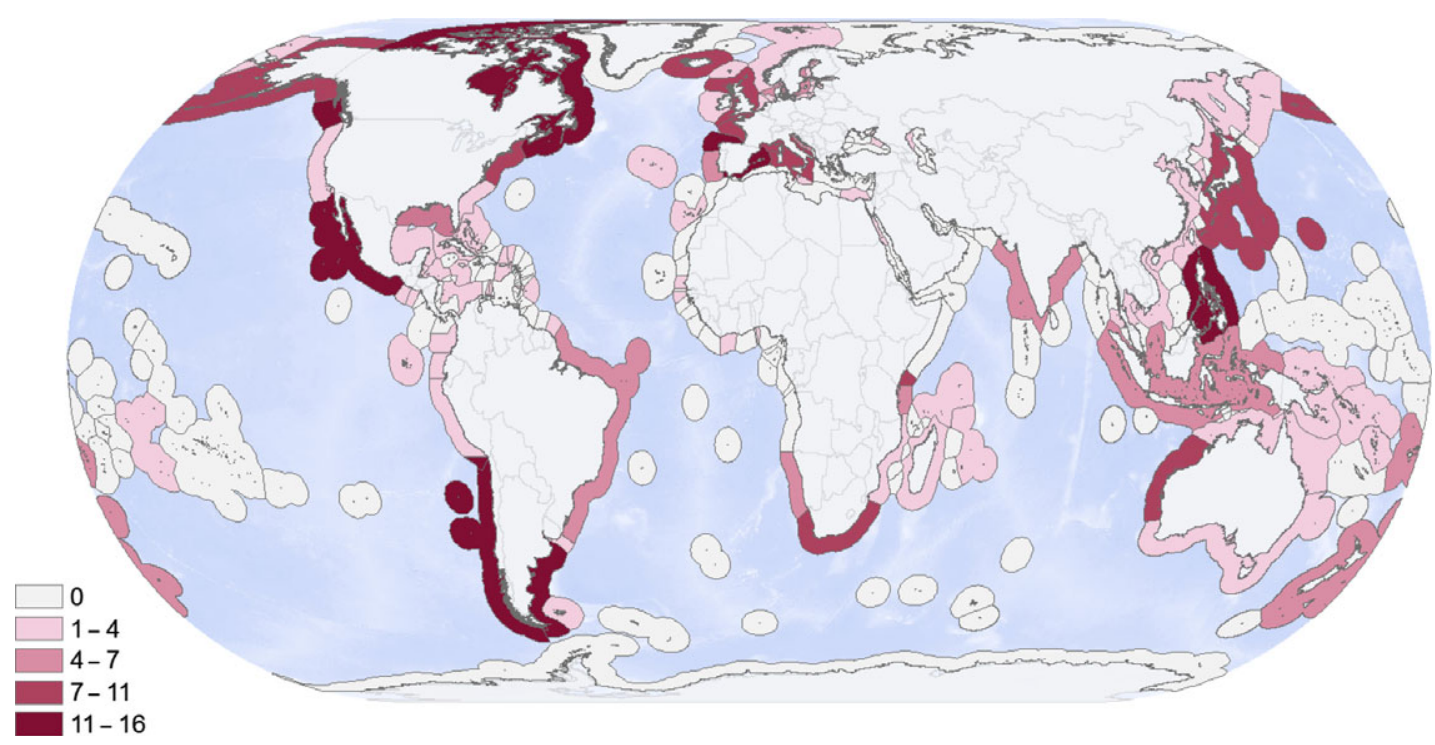

Figure 2 Map illustrating the number of studies per EEZ. Many regions where there are small-scale fisheries were not well represented in the literature. Generally, there were a greater number of studies in higher income countries. Studies that were transboundary were counted in each of the relevant EEZs. 
Table 4 Most common 'successful' management tools. The 'Percentage of cases used in combination' refers to the number of times the most frequently reported successful management tool for a region was used in combination with other management tools. Note that MPA 'success' in increasing abundance or improving status generally refers to finer scale changes within and immediately beyond MPA borders, whereas 'success' of other management tools can refer to impacts at the scale of the entire fishery or stock.

\begin{tabular}{|c|c|c|c|c|}
\hline Region & Total number of successes & Management tool & Success (\%) & $\begin{array}{l}\text { Percentage of cases } \\
\text { used in combination }\end{array}$ \\
\hline Eastern Central Pacific & 18 & MPAs & 67 & $17^{1}$ \\
\hline Indian Ocean & 30 & MPAs & 57 & $3^{1}$ \\
\hline Mediterranean & 29 & MPAs & 86 & $7^{1}$ \\
\hline North-east Atlantic & 51 & Gear restriction & 63 & 57 \\
\hline North-east Pacific & 17 & Quotas & 82 & $29^{1}$ \\
\hline North-west Atlantic & 16 & Quotas & 81 & 38 \\
\hline \multirow[t]{2}{*}{ North-west Pacific } & 9 & Limited entry & 78 & 78 \\
\hline & & Time-area closure & 78 & 78 \\
\hline \multirow[t]{2}{*}{ South-east Atlantic } & 1 & Quotas & $\mathrm{n} / \mathrm{a}$ & $\mathrm{n} / \mathrm{a}$ \\
\hline & 1 & MPAs & $\mathrm{n} / \mathrm{a}$ & $\mathrm{n} / \mathrm{a}$ \\
\hline South-East Asia/Oceania & 51 & MPAs & 49 & 12 \\
\hline South-east Pacific & 9 & Size limits & $56^{1}$ & $56^{1}$ \\
\hline Southern Ocean & 10 & Quotas & 60 & $30^{1}$ \\
\hline South-west Atlantic & 10 & Limited entry & 70 & 70 \\
\hline \multirow[t]{3}{*}{ Western Central Atlantic } & 19 & Gear restriction & 42 & 42 \\
\hline & & Quotas & 42 & 37 \\
\hline & & Time-area closure & 42 & 32 \\
\hline
\end{tabular}

MPAs, marine protected areas.

${ }^{1}$ Indicates that the management tool was used alone or in combination five or fewer times.

management tools, mobility, single or multispecies, ecosystem and management approach - there was an average $86 \%$ congruence. The greatest issues were in identifying the management approach. In particular, whether or not the tool was managed at the community level, national level or under co-management was sometimes unclear within the studied papers.

\section{Classification trees}

We used classification trees pruned to two nodes to determine which explanatory factors were most related to the successful or inadequate implementation of a given management tool. The relative success or inadequacy of a given management tool and associated factors can be estimated by looking at the ratios of inadequate to successful implementation provided along each branch of the tree. To this end, we computed classification trees with all factors, and with (Fig. 4) and without region (Figure S1). We confirmed the variables identified in the classification trees with binomial GLMs. Because of low sample size, classification trees and binomial GLMs could not be computed for time restrictions, TURFs or for time-area closures. For all computed trees, the binomial GLMs confirmed the first variable split in each tree (Table 5; Tables S3-S8). A factor level deemed significant in the GLM may not directly correspond to a combined factor level split in the classification trees because of differences in the two statistical approaches (see Supporting Information for further explanation).

With respect to limited entry, there were more successes reported overall than inadequate performances (55\% success). The first split in the classification tree was on the Human Development Index (HDI) scores, with more successes for medium or high index scores. At low HDI levels, there were more successes at medium and higher coastal population levels. For gear restrictions, there were more reported successes (68\% success) compared to those with inadequate performance. The first split was on coastal population, with greater success when the coastal population was at medium levels. When coastal population was either low or high, there was more success in benthic, demersal, 


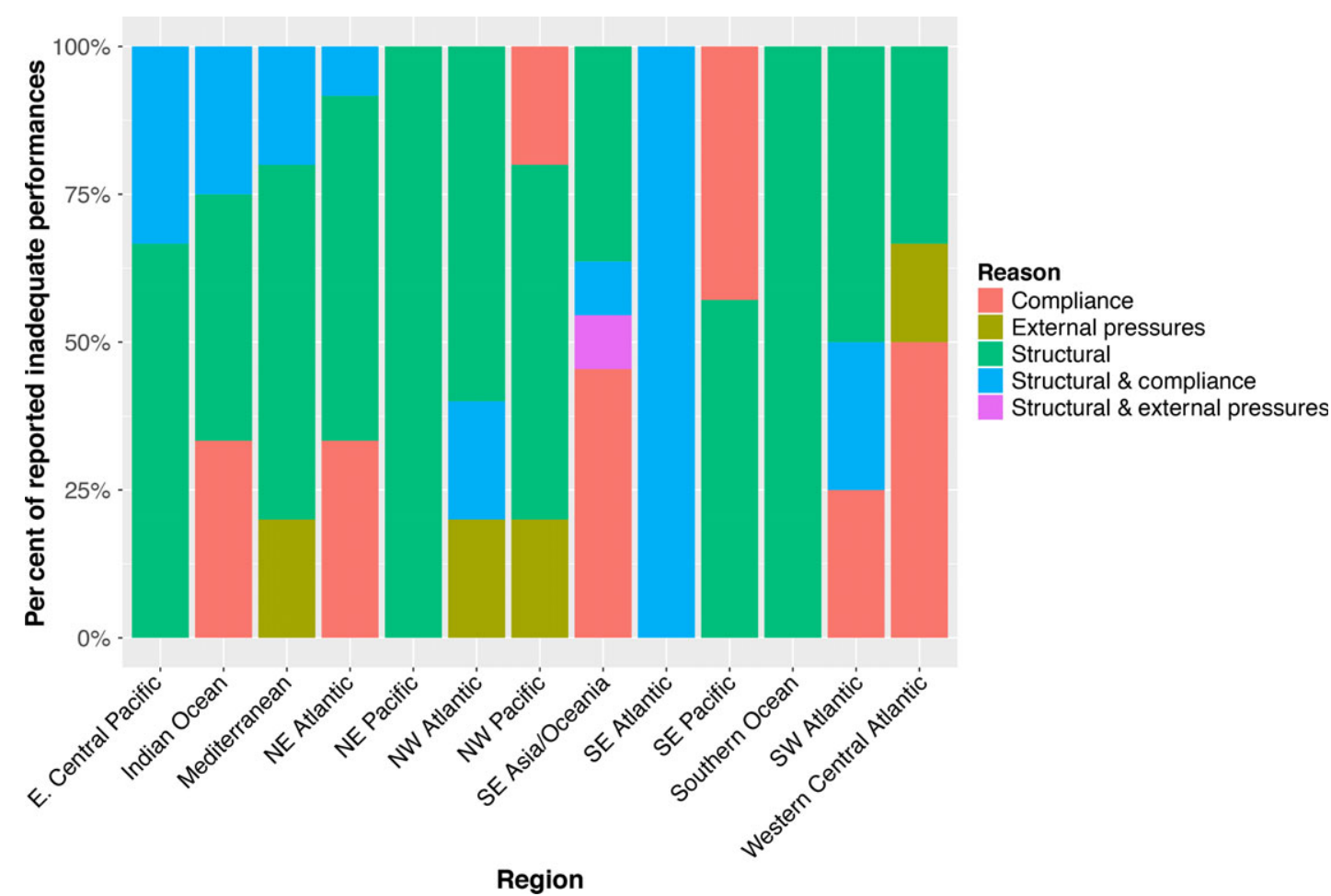

Figure 3 The distribution of reasons for inadequate performance as determined by the studies from each region. Overall, structural shortcomings in the design or implementation process were the most common reason for inadequate performance followed by compliance issues.

pelagic/demersal or reef habitats. The classification tree for quotas (i.e. catch shares and ITQs) looked similar to the tree for limited entry. Quotas also had more reported successes overall (68\% success) compared to those with inadequate performance. The first split in the tree was on HDI, with more successes at medium and high levels of development. At low HDI levels, there was more success at medium and higher coastal population levels. MPAs had a high reported success rate overall (77\% success), and generally more success when HDI scores were medium or high. At low HDI levels, there was more success when MPAs were used on their own and not as part of a combination of tools. Similarly, for time-area closures there were more reported successes (70\% success) and this management tool was generally more successful at medium or high HDI. At low HDI, time-area closures were more successful in benthic ecotypes or combinations of pelagic and demersal ecotypes. Size limits had more reported successes (76\% successful) at low and medium coastal population levels. At higher population levels, size limits were more successful when the fishery was targeting a single-species rather than multispecies fisheries.

When region was included in the classification trees, it was the first split defined in all trees, indicating that differences in contextual factors that vary by location are important. However, results from trees that included region were difficult to generalize and there was a higher degree of uncertainty because the sample size for each management tool by region was low (Figure S1).

\section{Expert-derived typology}

The expert typologies and literature review were largely complementary in that they considered distinct sets of potential factors that related to the performance of a given management tool. Several patterns emerged from our expert-derived typologies. In general, management tools within each category (i.e. input, output and technical measures, individually) tended to have similar patterns in the fishery characteristics that were important for management tool implementation, but the degree of importance of these characteristics for a 


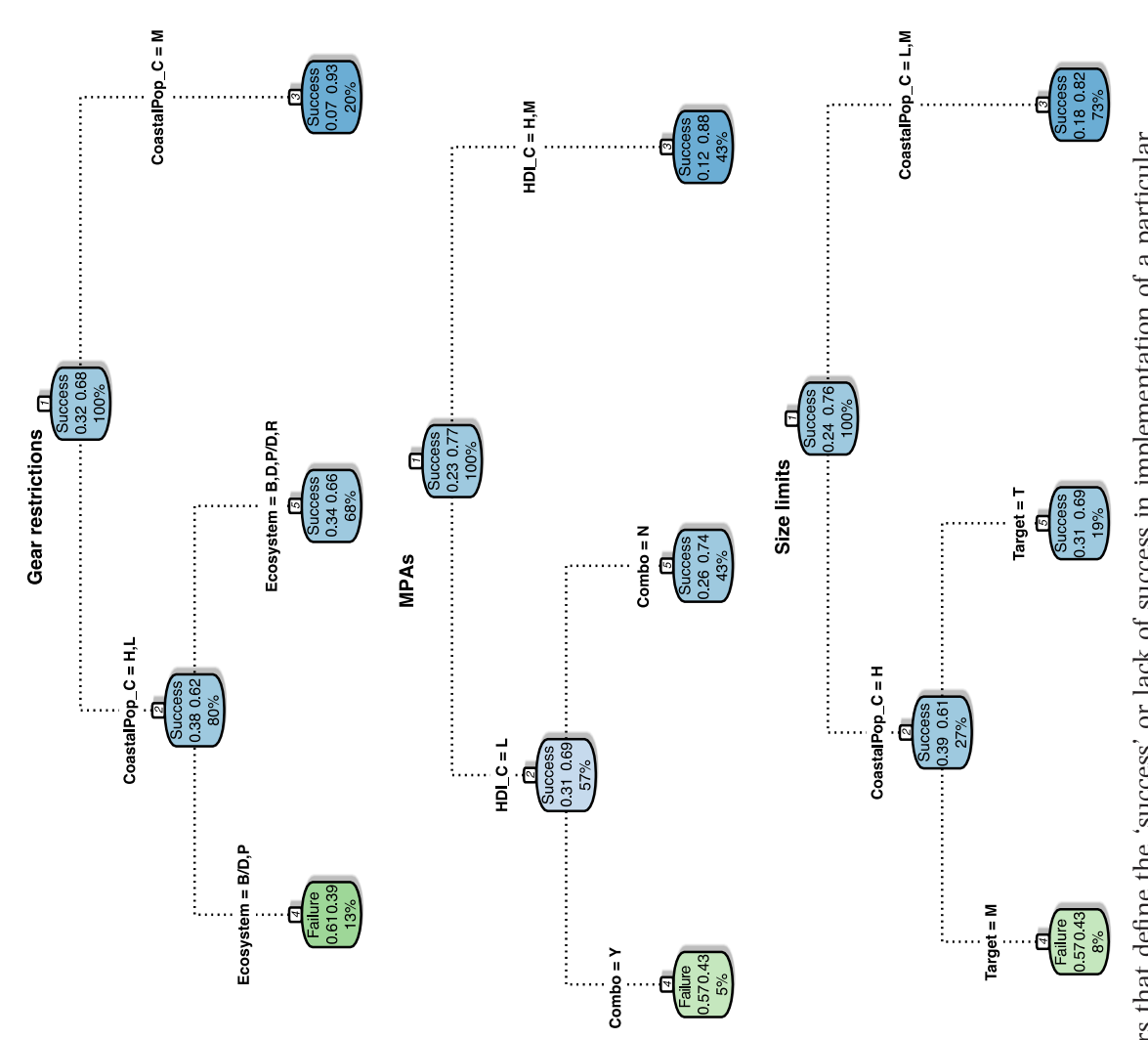

产密
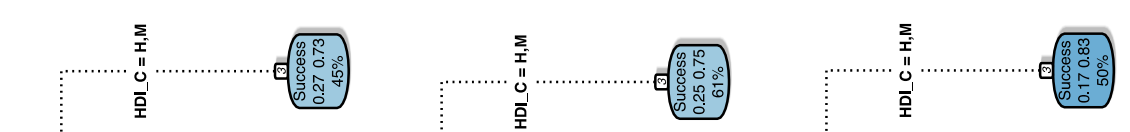

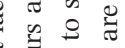
㽦

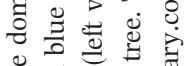
$\because \exists$

동

西 $\cong$

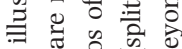

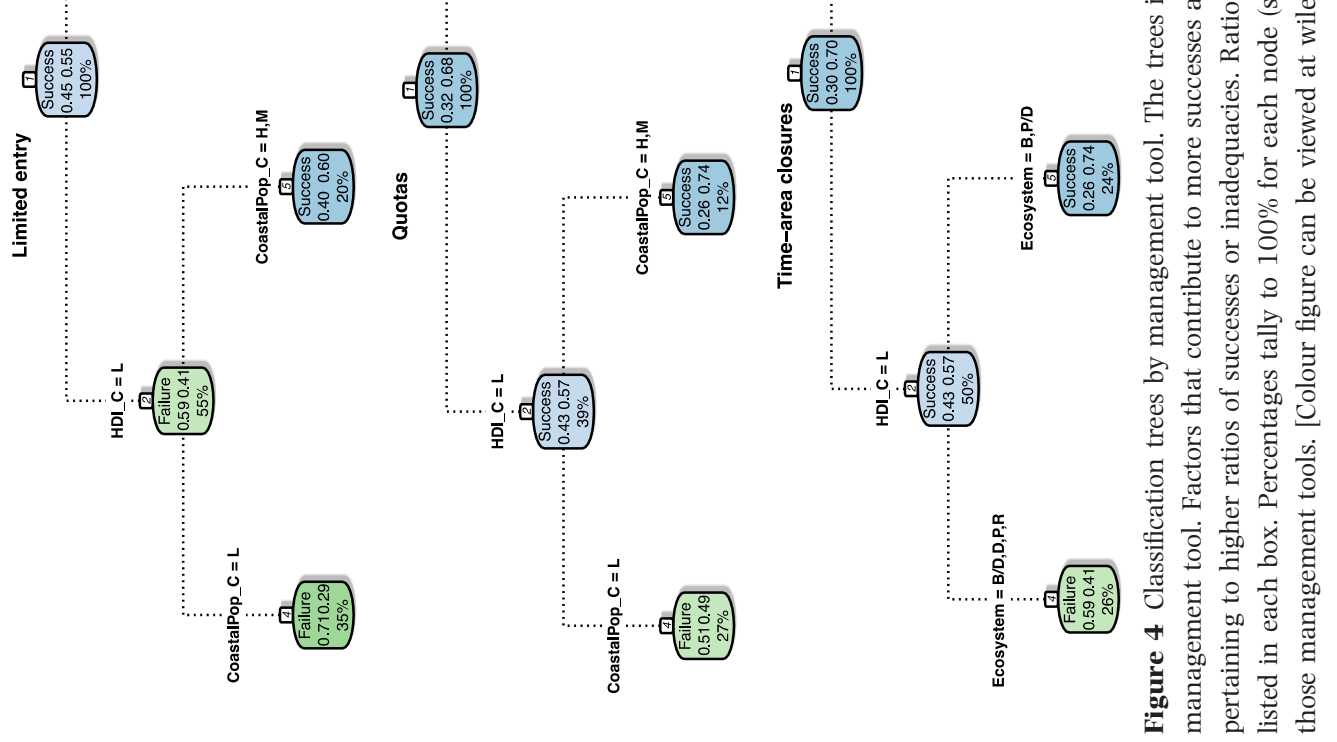


Table 5 Tools analysed with classification trees and the dominant factor for success when region was not included in the trees.

\begin{tabular}{ll}
$\begin{array}{l}\text { Management } \\
\text { tools }\end{array}$ & First node defined in classification tree \\
\hline $\begin{array}{l}\text { Limited entry } \\
\text { Gear restriction }\end{array}$ & $\begin{array}{l}\text { Human Development Index (medium to high) } \\
\text { Cuotas }\end{array}$ \\
$\begin{array}{l}\text { MPAs } \\
\text { Time-area } \\
\text { closure }\end{array}$ & Human Develation (medium) \\
Size limits & Coastal population (low to medium)
\end{tabular}

specific management tool varied (Table 6). In the typologies, experts identified whether a given factor was likely to be important for the successful implementation of a management tool. These classifications do not preclude use in other scenarios, for example input tools may also be useful in areas with decentralized landing sites, but centralized landing sites were thought to improve their chances of success. Input tools were generally considered more effective for multiple target species and species with high market value as well as in regions with high fisher density and centralized landing sites (Table 6). However, TURFs (an output tool) and MPAs and time-area closures (technical measures) were also considered effective for multispecies fisheries. Gear restrictions were the only input tool that experts determined could be effective for fisheries with high habitat degradation (Table 6). Size limits were distinctly different in that they did not closely follow the patterns of other tools (Table 6). Output tools were deemed particularly relevant for single-species fisheries (and in multispecies fisheries that use TURFs), stocks with high monetary market value, in fisheries with low fisher density, and with centralized landing sites (Table 6). Spatial technical measures were considered to be particularly effective for fisheries that have low-mobility species, consistent spawning seasons or locations and multiple target species. They were also found to be effective for fisheries that have high stock or habitat degradation (Table 6).

The group of experts generally believed that enabling conditions for input and technical tools track each other, with the magnitude of importance for different factors and the necessity for conflict management being the key differences (Table 7).
However, time-area closures were thought to rely more heavily on the existence of formal regulations than MPAs (Table 7). For output tools, the existence of formal regulations and legal regimes, the need for large amounts of information and national or municipal governance capacity were identified as key enabling conditions (Table 7). The presence and participation of fisherfolk organizations was considered highly important for the application of TURFs, but was also important for gear restrictions, limited entry, catch shares and ITQs, and MPAs (Table 7).

Experts identified several design considerations that could affect the success of a management tool (Table 8). These factors may affect the successful acceptance of the management tool by the community and the likelihood that they will comply with the regulations. The type of governance structure, a legitimate process of rule-setting and whether local communities were engaged in the design process were considered important design considerations for all management tools (Table 8).

\section{Discussion}

Together, results from the literature review and expert-derived typologies can help managers identify which management tools may be more effective given the characteristics of their fishery. Because the literature review likely reflects some biases in reporting and geography in the available studies, it can only give a general indication of which factors may drive management tool performance in a biological context. Many regions where small-scale fisheries are known to be important were not well represented in the literature and key factors including social, economic and cultural metrics, which may be more relevant to defining 'success' in a small-scale fishery, were not reported. For this reason, we also developed expert-derived typologies to bridge these gaps by examining a broader suite of fisheries characteristics, enabling conditions and design considerations to determine which factors may be most important for the effective implementation of different management tools (Tables 6-8). All management processes will require learning and adaptation to further improve underperforming management scenarios or the development of new management approaches. Ideally, our results should be used in the design process to help to catalyse innovation. 


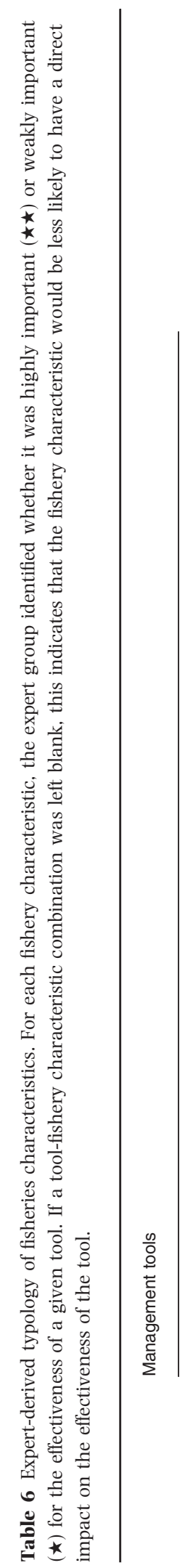

14

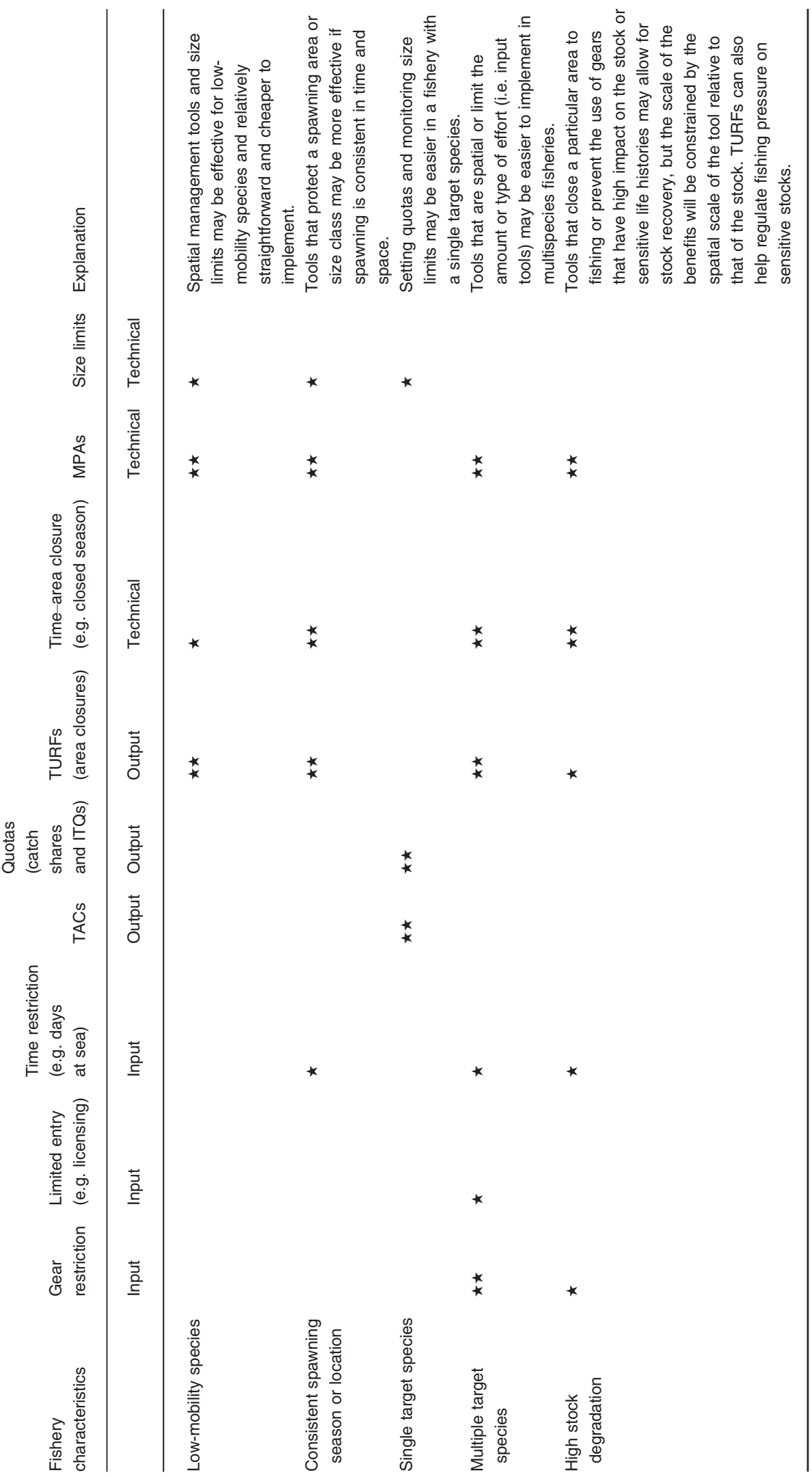

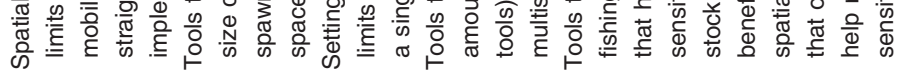

C 2016 John Wiley \& Sons Ltd, FISH and FISHER IES 


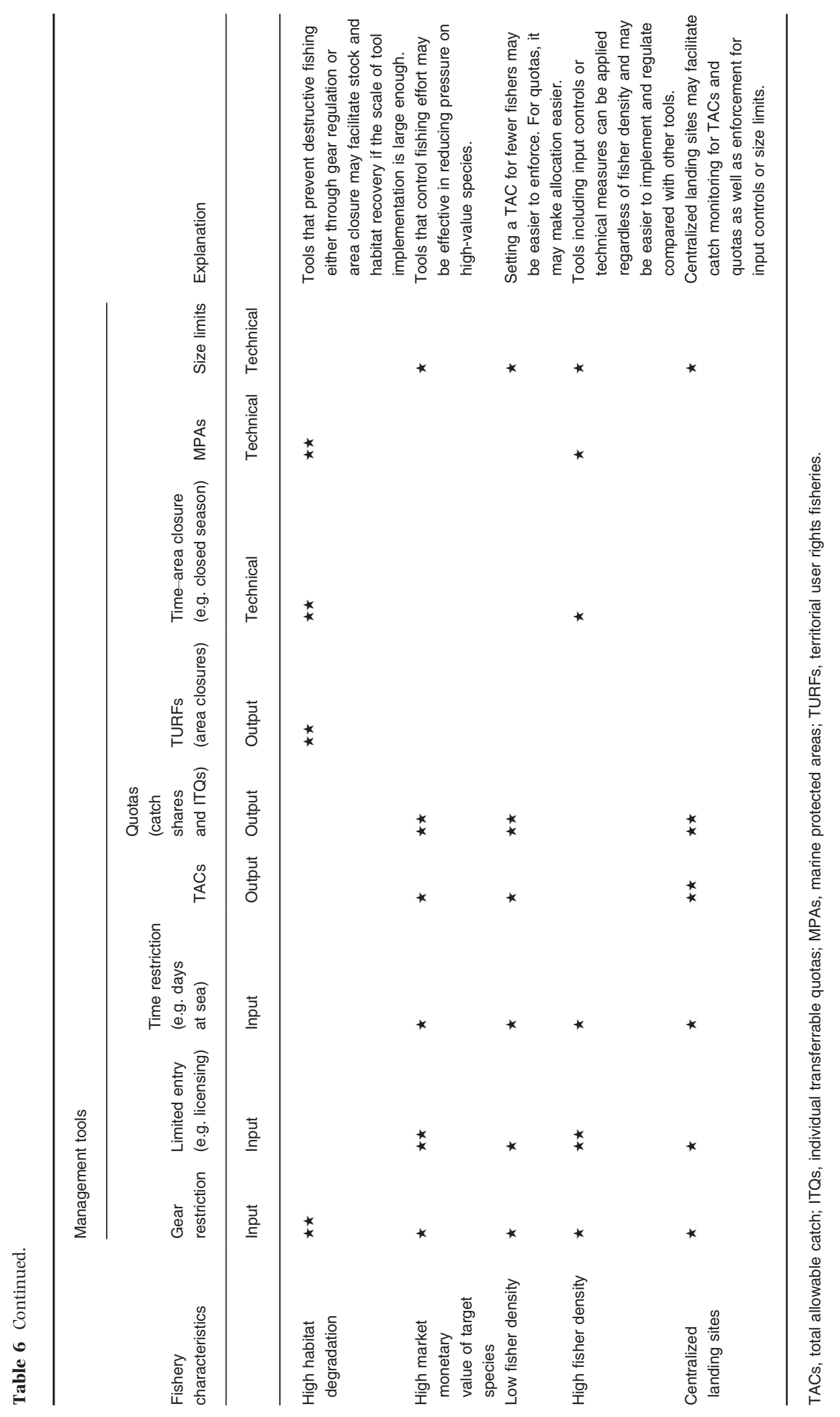




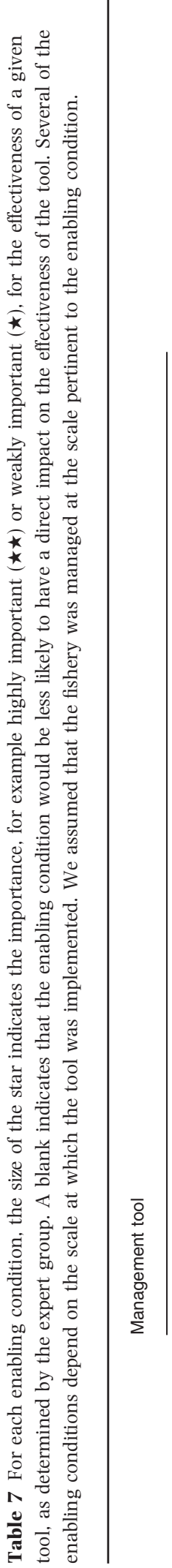

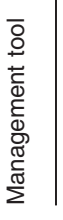

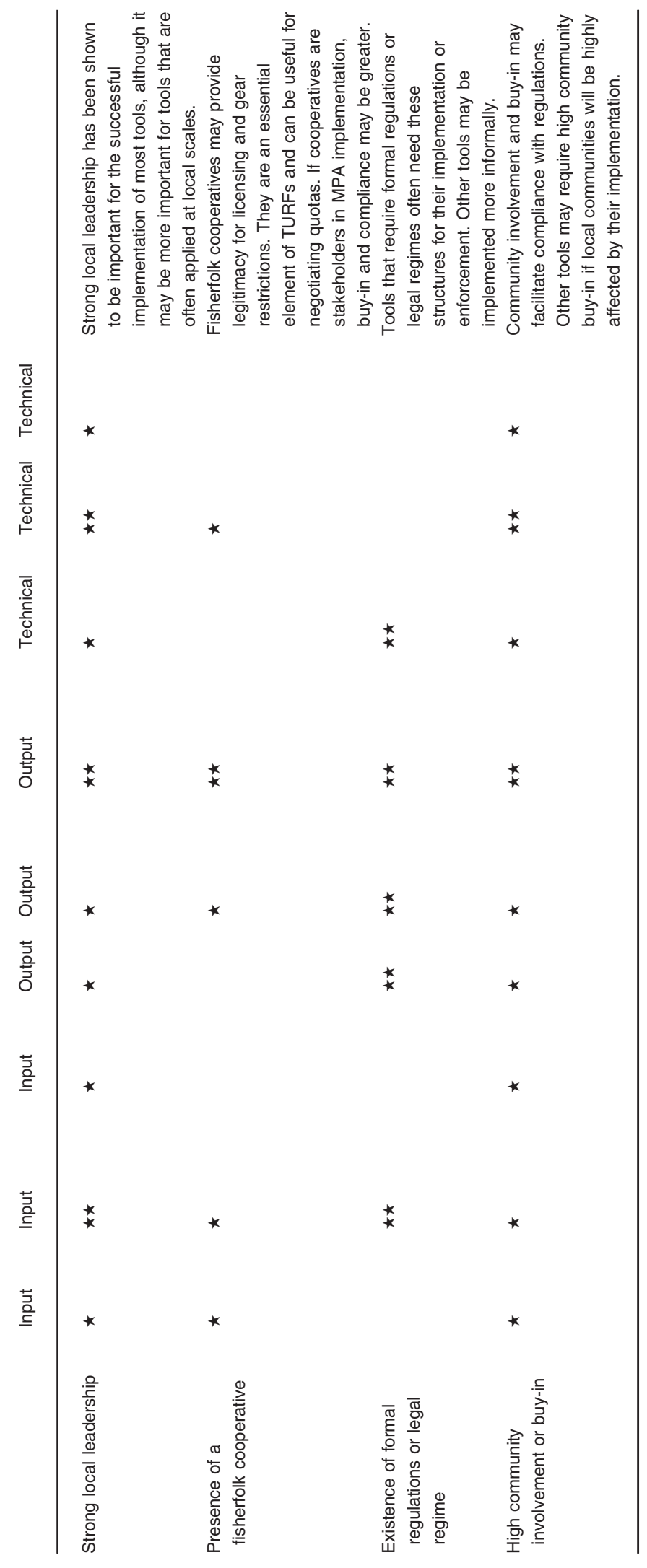




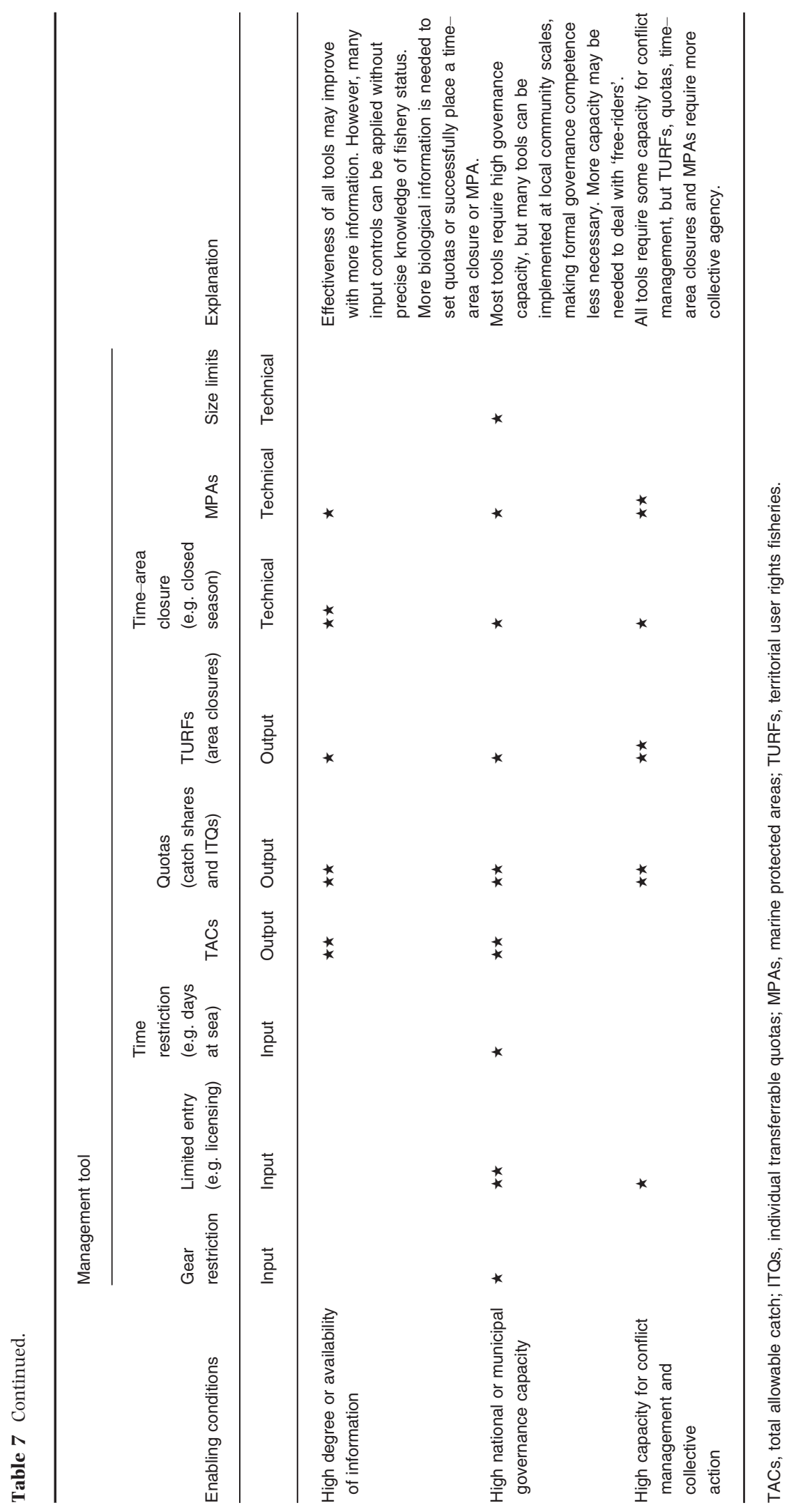




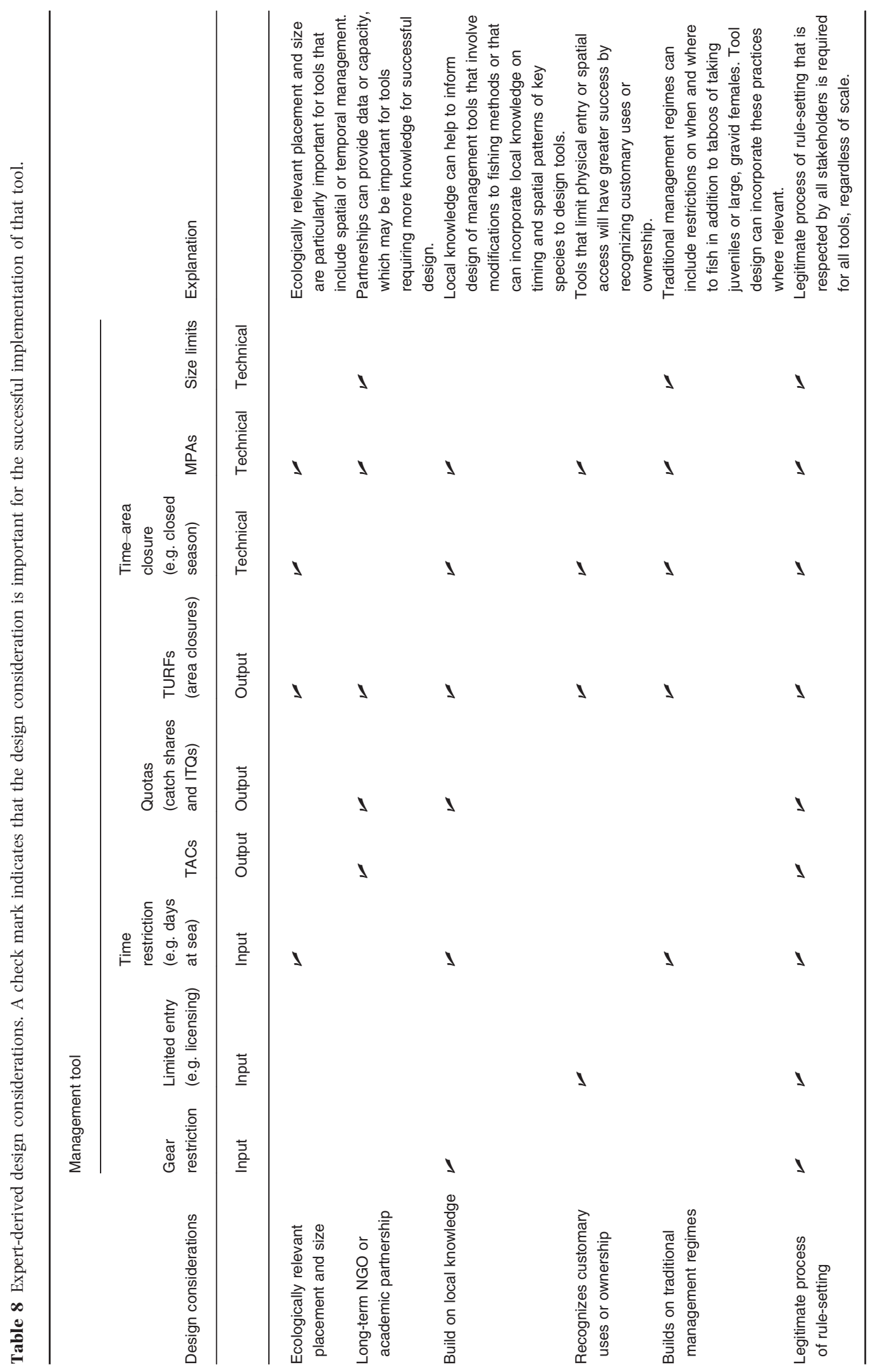




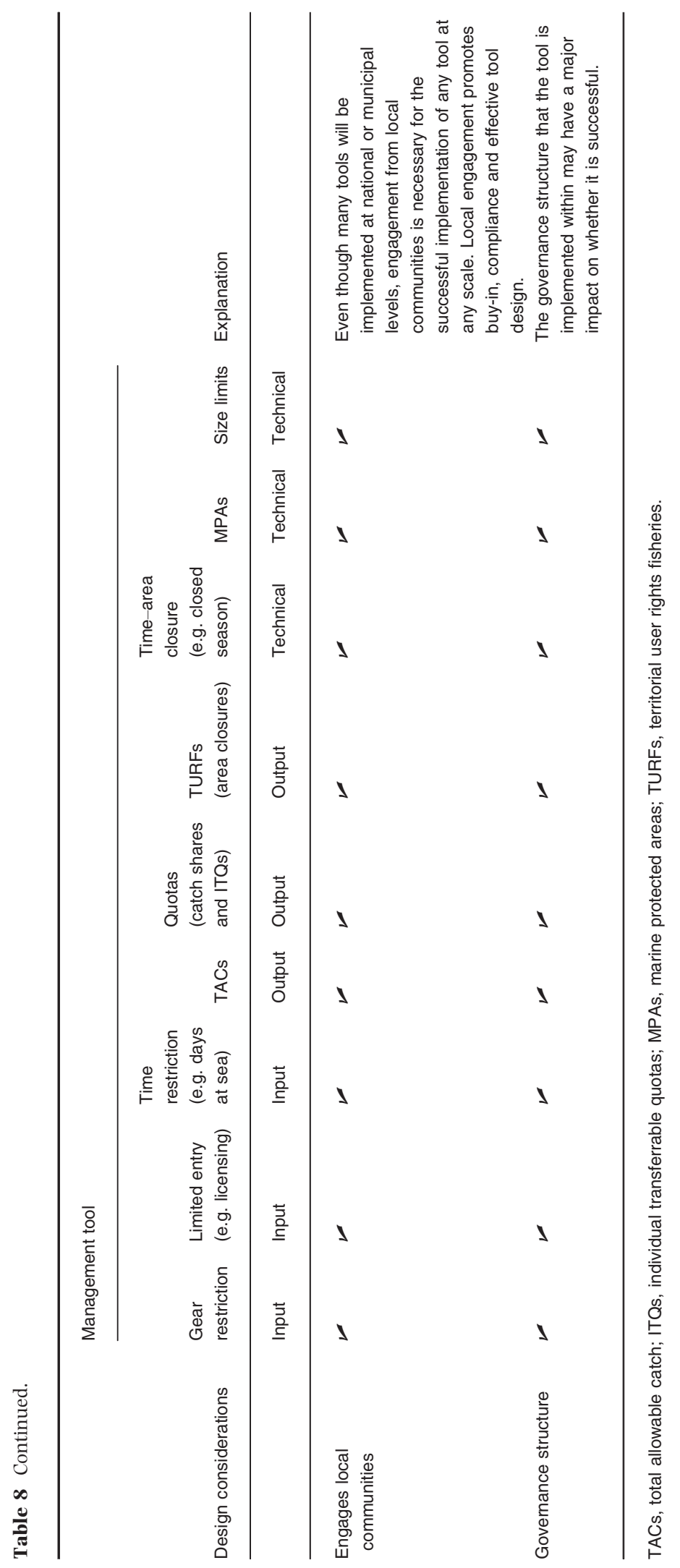


From the literature review, we found that the most commonly implemented management tool in a region was also generally judged the most successful in biological terms (Tables 4 and S2). Exceptions to this general result were found in the Eastern Central Pacific where gear restrictions were the most commonly reported tool, but MPAs had higher rates of biological success, and the North-east Atlantic where time-area closures were more common, but gear restrictions had higher rates of biological success (Tables 4 and S2). The review also indicated that implementing multiple management tools can lead to greater success. Several studies have found similar patterns with combinations of different tools (Stefansson and Rosenberg 2005; Mahon et al. 2008; Little et al. 2011; Ovando et al. 2013). Some of the key successful combinations in our study were the use of TACs with ITQs and limited entry; the use of gear restrictions with TACs, time-area closures and MPAs; and the use of MPAs with management tools that restrict effort.

There are likely to be several distinguishing characteristics between what is most successful in large- vs. small-scale fisheries. While we could not explicitly analyse differences between large- and small-scale fisheries due to small sample size, our literature review suggests that the frequency with which a tool was implemented varied between large-scale vs. small-scale fisheries, as defined by Allison and Ellis (2001). Frequency of tool implementation may relate to the biology of the fishery itself or the governance, social or cultural context of the fishery. In our literature review, MPAs and TURFs were implemented $60 \%$ and $70 \%$ of the time in small-scale fisheries, respectively, whereas quotas and TACs were implemented in large-scale fisheries nearly $85 \%$ of the time. Therefore, lessons learned with respect to these tools are largely associated only with the scale of fishery within which they were applied. Much may still be learned about what may be needed for success if they are to be applied in other contexts than what is reported. In addition, large- and small-scale fisheries may have different metrics for success beyond the biological metrics that we studied, although these will vary by fishery (Béné et al. 2006, 2010; Allison et al. 2012; Weeratunge et al. 2013; Hicks et al. 2014). For example, large-scale fisheries outcome objectives may relate to profitability and efficiency (Sethi et al. 2010) whereas small-scale fisheries metrics for success beyond biological characteristics may be more related to aspects of human well-being including but not limited to issues of employment, identity, economics and safety (Allison et al. 2012; Weeratunge et al. 2013, FAO, 2015). Consistent reporting and large-scale assessments of social and economic metrics of success in small-scale fisheries will be needed to gain a better understanding of tool performance in an exclusively small-scale fisheries context.

There are many potential reasons for the pattern of greater success in more frequently implemented tools. Positive reinforcement from the successful implementation of a tool can often spur an increase in the implementation of that tool as it becomes more familiar (Wejnert 2002). In addition, a success or handful of successes with a particular tool or tools may catalyse greater investment in the resources necessary for further successful implementation. When a tool is selected, the degree of financial and human resources and planning can all influence its success, making it hard to distinguish whether the tool itself was intrinsically better or whether the resources dedicated to it increased the chances for success. In some cases, the existing enabling conditions are barriers for the implementation of a particular tool in some geographies (Diekert et al. 2010). Finally, reporting biases in the literature may also contribute to the pattern of greater successes amongst the most frequently implemented tool(s) in a certain place.

In fact, the classification trees based on the literature review results suggest that some extrinsic factors including the level of human development as measured by the Human Development Index (HDI) and the density of the coastal population have a relatively stronger relationship to the performance of most of the management tools than other factors examined here. In general, limited entry, quotas, MPAs and time-area closures were more successful when the HDI was at medium to high levels. This suggests that countries or regions with the resources to invest in fisheries management and enforcement were more likely to report successful tool implementation. This pattern may also reflect other differences such as scientific and administrative capacity and rule of law in a particular fishery, but our literature review could not systematically assess such factors.

Size limits were more successful when coastal population levels were low to medium. 
Monitoring can be more challenging in fisheries with many participants (Anderson and Seijo 2010), so lower human population levels could increase the potential for success. Also, size limits have been shown to alter population structure because they force fishermen to select a particular component of the population, for example adults over juveniles (Borrell 2013). This may reduce the productivity of the population if larger individuals have higher reproductive output. Therefore, in areas with lower coastal populations and possibly fewer fishery participants, size limits may impose less pressure on significant components of the population. Difficulty in controlling access at high coastal population levels may also be an issue with gear restrictions. However, our results suggested that gear restrictions were also less successful at low coastal population levels, which may indicate that other factors we did not assess, such as the vulnerability level of the species being fished (Brewer et al. 2013), may also play a role in the successful implementation of gear restrictions.

The degree of access limitation may be important for the successful implementation of many tools, although it was not something we were able to consider in our analysis because it was not often reported in the literature. Management tools can be overwhelmed by increasing numbers of fishers (Geheb and Binns 1997) or a lack of secure fishing rights (Committee on World Food Security and FAO, 2012; Grimm et al. 2012, FAO, 2015). Critically, the nature and distribution of fishing rights can also have strong effects on buy-in and compliance (Allison et al. 2012). Fishers who do not have secure rights may be unable to obtain the benefits of compliance directly, because they accrue to the entire fleet. A recent study of the importance of this phenomenon to conservation performance found a strong positive relationship between the presence of spatial harvesting rights (TURFs) and the establishment of MPAs by fishing cooperatives (Ovando et al. 2013). At the extreme, inequitable distribution of fishing rights or systematic barriers to access and benefit sharing by poor and marginalized groups may spur conflict (Pomeroy et al. 2011; Allison et al. 2012).

Experts identified strong local leadership, community involvement or buy-in and municipal or national governance capacity as being particularly important for the implementation of every management tool (Table 7). Even when management tools are implemented nationally, local leadership can be critical for helping to ensure a successful implementation process, including buy-in from the community (Gutierrez et al. 2011). High national or local governance capacity refers to the ability of the relevant level of government to design, implement and enforce a management tool (Fanning et al. 2013). Many management tools can be implemented successfully at the local scale with more informal governance structures. However, at the local or community scale, adequate capacity is still required to design a management approach, secure local buy-in and enforce regulations. In some cases, though, informal regulations may result in confusion or cause conflict with more formal national and local regulations. Clear communication and coordination between different authorities on formal and informal regulations is essential.

Many of the reasons that management tools were deemed inadequate were related to 'structural' factors, which included both poor design and implementation (Fig. 3). Structural issues were most common in fisheries in the Northern Hemisphere, potentially because output controls, which require more thorough monitoring and enforcement, were applied there more often. Compliance failures were common in the regions where MPAs are most frequently applied. Understanding compliance necessitates a broader understanding of the actors and the context, but compliance failures broadly reflect a failure in the attitude towards compliance (e.g. due to a failure to obtain stakeholder buy-in) or the absence of an effective compliance management or enforcement strategy (McClanahan 1999; Arias 2015). Inadequacies associated with using MPAs as a fisheries management tool can also be exacerbated by a failure to successfully manage surrounding fisheries (Mesnildrey et al. 2013). Interestingly, though, external pressures were not commonly listed as a reason for poor performance. However, this result may reflect the use of narrow success metrics (e.g. a biomass increase within MPA boundaries) or the difficulty in monitoring pressures like climate change, cumulative human impacts or illegal fishing (Fig. 3). The exception was for South-East Asia/Oceania where climate change-related factors were listed as the main drivers, potentially due to the sensitivity of relatively well-studied coral reef fisheries to losses in coral cover as a result of climate change impacts (Bruno 
and Selig 2007; Munday et al. 2008; Allison et al. 2009).

Fisheries management tools are often implemented with different goals and at different scales (Table 2). A successful outcome for one management tool may not be realized at the same spatial scale as the outcome of another, depending on how it is implemented. For example, spatial scale may be a particularly important consideration when assessing area-based management tools such as time-area closures, MPAs and TURFS. Biological performance indicators such as fish abundance, density and size may increase within and near borders, but fishery-level outcomes will depend on the characteristics of the species involved, the scale of implementation, the effective use of other management tools and whether managed areas are networked. No-take marine reserves can have different performance outcomes than multiuse MPAs (Lester and Halpern 2008; Sciberras et al. 2015) and factors of success may be different as well. In addition, the fishery characteristics themselves affect the performance of management tools at various scales. For example, the Human Development Index was an important factor for limited entry, quotas, MPAs and timearea closures, but it is calculated at a scale beyond which most fisheries management tools are implemented. Overall, it is important to recognize that the factors that affect the successful implementation of a management tool may not always be at the management scale. Therefore, it is prudent to consider the wider socioeconomic and governance context within which a tool is implemented (Andrew et al. 2007; Ratner et al. 2012).

The appropriate temporal scales for achieving and evaluating success also differ by management tool and conditions, including the state of the fishery when management is initiated. In addition, because we defined success in biological terms, we were not able to determine whether a management tool was successful in increasing seafood availability to local communities or in improving livelihoods. If designed and implemented with a sole focus on increased biomass, an MPA or other management tool that did achieve biological 'success' might still be viewed by the community as inadequate if it reduced well-being by impairing access to seafood or income. In addition, our analysis does not preclude a change in success rates for a given tool over time.

\section{Patterns within management tool categories}

We examined patterns within our three major tool categories in both the literature and expert typologies, because they often exhibited similar factors relating to their success. It should be noted that when we discuss success within a given tool category, we are not comparing across tool categories. In addition, application of a tool in a context other than the one we highlight does not preclude success in other circumstances.

\section{Input controls}

Input controls were used relatively frequently in most regions, with limited entry and gear restrictions used more often than time restrictions. Limited entry (e.g. licensing) was found to be more successful in countries where HDI was higher (Table 5). Gear restrictions were generally more successful when used in combination with other tools (Table 4). The typologies generated by the experts generally showed that input tools would be most effective for multiple target species, species with high market monetary value, centralized landing sites, and could be applied at either low or high fisher density (Table 6).

Input controls have been thought to be easier to implement and less costly to monitor and enforce than output controls (FAO Fisheries Department, 2003). However, input controls can require administrative and monitoring capacity to determine how much effort is really being exerted by each fishing vessel and catch per unit effort may not be constant if the stock size varies (FAO Fisheries Department, 2003). More recent research has shown that because vessels can vary greatly in their efficiency and technology, quantifying and managing effort can be challenging (Villasante and Sumaila 2010). Effort creep, increased fishing effort that reduces the management effect of the input control, may limit input tool effectiveness (Kompas 2005; Grafton et al. 2006). For example, gear restrictions like trawl bans have been effective in protecting benthic invertebrates and lower mobility fish in certain contexts (Rice 2006), but less so in others because of gear substitutions or modifications (Kompas et al. 2004). The typologies generated by the experts suggest that the existence of formal regulations may be important for limited entry, but less so for gear or time restrictions (Table 7), which can be applied informally through the use of customary behaviours (e.g. 
particular gears are used or avoided, NickersonTietze 2000) and cultural taboos (e.g. no fishing on a particular day, Harkes and Novaczek 2002).

\section{Output controls}

Expert typologies suggested that TACs as well as quotas (i.e. catch shares and ITQs) would be easier to implement in fisheries that have a single target species, relatively low fisher density and centralized landing sites because monitoring catch levels would be easier under those conditions (Table 6). Monitoring is a key challenge for output controls (Hersoug and Paulsesn 1996, FAO Fisheries Department, 2003). In spite of those challenges, output controls were implemented with nearly equal frequency in targeted and multispecies fisheries (Table S2). The use of TACs on their own was often found to be ineffective, a result that is also highlighted in the literature (e.g. Baudron et al. 2010; Huang and $\mathrm{Ou}$ 2012). This was likely due to the fact that a 'race to fish' may be induced if TACs are implemented in the absence of a governance structure or economic instruments that counteract incentives to maximize catch. However, in combination with quotas and limited entry, TACs had more success (Table 4; e.g. Orensanz and Seijo 2013). Additionally, TACs were more successful in fisheries operating on benthic or demersal habitats, although the mechanisms driving their success were not clear from our review.

Ensuring high compliance with TACs may require expensive monitoring and control systems, and significant administrative capacity. Catch share and ITQ programmes can ameliorate these impacts by allowing fishers to transfer quotas and legally land fish that are caught incidentally, reducing discard rates (Grimm et al. 2012). The expert typologies found similar enabling conditions for quotas and TACs. However, the presence of fisherfolk cooperatives and the capacity for conflict management were thought to be important when negotiating allocations for quotas in small-scale fisheries (Table 7). Output controls that are implemented in the absence of a governance structure that encourages collective action or stewardship behaviour may not address race-to-fish (derby) dynamics, which can result in lower profits, high discards, habitat impacts and other adverse effects (Turner 1997; Hoggarth et al. 1999).

Although we considered TURFs to be output tools because they can restrict the amount of fish that an individual can take, they also control where those resources may be harvested (Finlay et al. 2013). In addition, they are often considered to be a form of rights-based management because they are predicated on secure and exclusive rights to harvest in certain areas. As a result, TURFs tended to have more in common with other spatial management tools and were considered to work best in fisheries with low-mobility species, consistent spawning seasons or locations and multiple target species (Table 6). TURFs also had more in common with other output tools in terms of enabling conditions (Table 7).

\section{Technical measures}

The expert typologies suggest that MPAs and time-area closures would work best in fisheries with low-mobility species, consistent spawning seasons or locations and multiple target species (Table 6). Spatial management tools were also deemed to be reliant on ecologically relevant placement and size (Table 8). This reflects the fact that closed areas may work best in some situations when they are part of a network and when they are linked to broader-scale fishery management measures. Size limits had similar characteristics, but were more effective for single target species and with centralized landing sites (Table 6). Timearea closures and MPAs were most successful when used in countries with relatively high HDI values (Table 5, Fig. 4), potentially reflecting the capacity needed to design and monitor them (O'Keefe et al. 2013).

Successful outcomes for MPAs are often restricted to the rebuilding of fish density and agestructure within or nearby MPA boundaries, that is localized improvements, rather than at the scale of whole fisheries or stocks. For example, MPAs were often reported to be successful in the Mediterranean (Table 4), but this high rate of reported success of MPAs should be interpreted with caution due to the fact that the scale of these impacts do not appear to have fishery-wide effects and many Mediterranean fisheries are overfished (Colloca et al. 2013).

In general, experts felt that more information about stock structure, migratory patterns and other life-history traits would need to be available when implementing a time-area closure vs. an MPA because a time-area closure relies on understanding both temporal and spatial dynamics (Table 7). However, the temporal flexibility of a time-area closure may have greater appeal in 


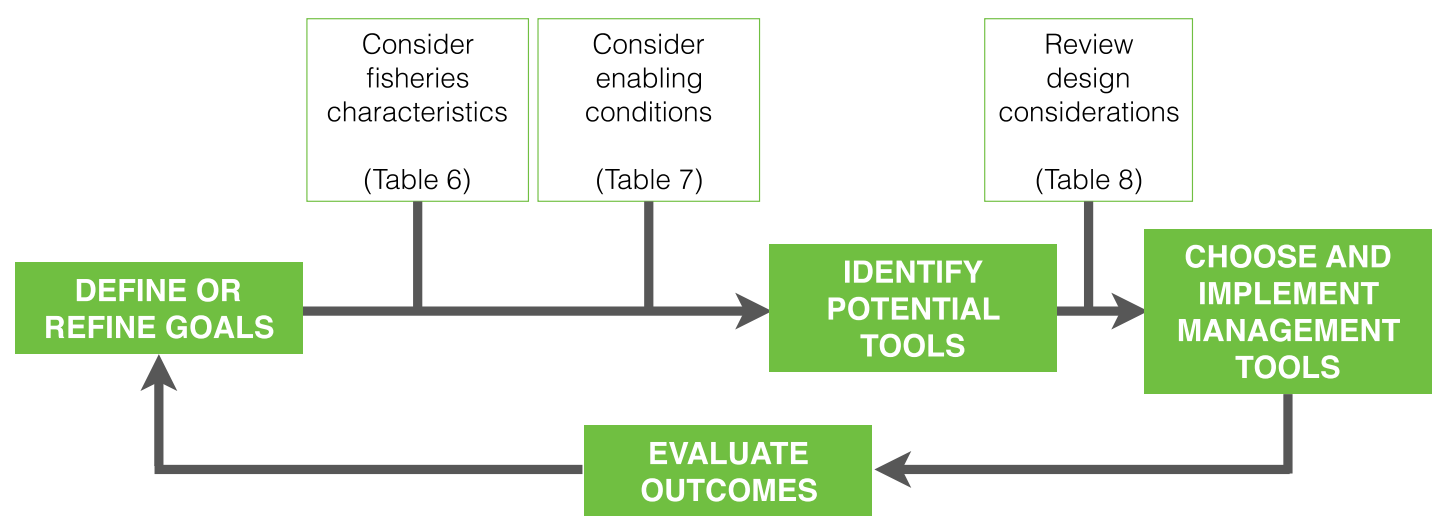

Figure 5 Overall management design process for selecting management tools. The typologies can be used once fishery characteristics and enabling conditions for a given fishery are identified. The process is a feedback loop as fishery characteristics and enabling conditions can change.

areas where there is a desire to maintain some fishing. Time-area closures were also thought to rely more on the existence of formal regulations or legal regimes than MPAs, but this finding is likely highly dependent on the governance scale of the MPA and whether it is a no-take zone or a more complex multiuse area. The degree to which stakeholders acknowledge the legitimacy of and comply with informal regulations is also an important consideration. Time-area closures are temporary, so the need for conflict management was thought to be less than what would be needed for permanent closures like TURFs and MPAs (Table 7).

Integrating 'lessons learned' into the process of determining a fisheries management approach

Lessons from our analyses can be applied within a broader management design process (Fig. 5). During the management design process (Fig. 5), stakeholders can identify the applicable fishery characteristics and enabling conditions for their fisheries and compare them to the typologies to determine which tools or sets of tools may have the greatest likelihood of biological success. For example, if we consider a hypothetical coral reef fishery that targets multiple reef-affiliated species with relatively low mobility and consistent spawning seasons, potential tools would include TURFs, time-area closures, and MPAs, or possibly combinations of those tools. A hypothetical fishery for highly mobile pelagic species with high market value and low fisher density could consider a different suite of potential tools that may include gear restrictions or quotas. Stakeholders would also need to consider the suite of design considerations applicable to a given fishery. We designed our typologies to be used as guidance for deliberation within the context of an overall multistakeholder management process (Fig. 5).

How this process is conducted may be a crucial factor in determining whether or not a tool will be effectively implemented. Experts felt that three design considerations were particularly important for the successful implementation of any tool - a legitimate process of rule-setting, an understanding of the governance structure that the tool would be embedded within and a process that engages local communities (Table 8). A rule-setting process was considered essential because it provided the foundation for any subsequent buy-in (i.e. stakeholders must feel that the rule-setting has been legitimate and responsive to local conditions) and compliance (i.e. everyone understands what the rules are). The type of governance structure was also thought to be important because it may have a strong impact on whether the tool or set of tools can be successfully implemented or not. For example, because governance structure may affect access rights, the ability to engage in collective negotiation or action and capacity for stakeholder engagement, it would have major impacts on the successful implementation of many output tools. Additionally, because governance may affect compliance, it could also have major impacts on the success of input controls and technical measures.

Engagement of local communities was also viewed as crucial, regardless of the scale of 
implementation, because lack of engagement will likely result in poor buy-in, stewardship and compliance with regulations, which can be especially problematic in fisheries lacking high enforcement capacity. In other words, even a top-down management tool implemented at the national scale should engage local communities because doing so would improve compliance (Walmsley and White 2003). Engaging local communities may also help to guide a more effective design of the tool itself. Fishers or stakeholders who have been affected directly by the implementation of tools that have been successful or inadequate can provide valuable perspectives on how to better design tools. Several management tools, including MPAs, TURFs and size limits, can build on local knowledge about the fishery, incorporate customary uses or ownership or build on traditional management regimes. (Table 8; Harkes and Novaczek 2002). Engagement with a non-government organization (NGO) or academic partner can also help to build local management capacity or provide data that can be used in the design of the tool (e.g. the size and placement of an MPA).

Decisions about which tools may work best in a given place can be improved with the use of additional quantitative and qualitative data. We generally only considered English-language studies here, but a systematic review that includes other languages may provide context for other geographies. Our literature review and classification tools indicated that the geographic location where a tool is implemented appears to be an important determinant of its success, but our sample size for understanding how region affects other factors was too small to be informative. If more studies can be included, we may be able to get a better understanding of the role of place in determining the success of a given tool. Additionally, although we explored which combinations of tools were implemented together and whether they were successful, we could not systematically determine which factors led to their success. We were also unable to fully consider combinations of tools in our expert-derived typologies, but their success suggests this is an important area of future research.

Because of the structure of our analysis and current data limitations, we were not able to assess 'success' beyond biological criteria from the literature, compare performance across tools or delve as deeply into specific issues for small-scale fisheries with respect to tool implementation. Although social and economic criteria are important for assessing the degree of management success in both large-scale and small-scale fisheries, they are perhaps even more exigent for assessing smallscale fisheries where aspects of human well-being are so closely tied to how management is implemented. If more data on other success metrics become available, a broader consideration of 'success' that includes more dimensions than the biological ones considered in our literature review may be possible. In addition, the inclusion of a broader set of criteria in assessing performance such as those outlined in the FAO's Voluntary Guidelines for Securing Sustainable Small-Scale Fisheries (2015) would allow for greater assessment of poverty, food security and other socialeconomic dimensions. A greater focus on the specific set of issues in small-scale fisheries would be improved by on-the-ground surveys with communities, managers, civil society organizations and fishers' organizations as well as further expert elicitations to get a broader range of perspectives in different regions. A comprehensive framework such as this would need to be created before the relative utility of these tools in various contexts can be fully understood, and the trade-offs amongst them appreciated, particularly in smallscale fisheries.

Nonetheless, our results can inform several key stages of the management design process. We built on existing work by considering a full suite of management tools and systematically assessing their biological performance in the literature. We also considered a much wider array of factors that may contribute to successful management as defined through expert input. Using the complementary literature review and expert typology approaches, our results can serve as an initial step in identifying a potential suite of tools to consider for a particular fishery, their associated enabling conditions, and which combinations of tools are likely to be particularly effective in different situations. The findings presented here may also provide guidance in the learning and adaptation that is part of any management process. Expert-derived design considerations can be used to help guide the successful development of a management plan and implementation of management tools. Together, our results can help managers learn from past experiences so that they can increase the likelihood of effective management decisions in their own fisheries. 


\section{Acknowledgements}

This research was generally supported by a grant from the Rockefeller Foundation. We thank Brian Crawford, Thomas Grasso, Jessica Landman, Doug Rader, Cristina Rumbaitis del Rio and John Thomas for their valuable input. SV acknowledges the financial support from the European Commission (Europe-Aid) and the Norwegian Research Council.

\section{References}

Allison, E.H. and Ellis, F. (2001) The livelihoods approach and management of small-scale fisheries. Marine Policy 25, 377-388.

Allison, E.H., Perry, A.L., Badjeck, M.C. et al. (2009) Vulnerability of national economies to the impacts of climate change on fisheries. Fish and Fisheries 10, 173-196.

Allison, E.H., Ratner, B.D., Åsgård, B., Willmann, R., Pomeroy, R. and Kurien, J. (2012) Rights-based fisheries governance: from fishing rights to human rights. Fish and Fisheries 13, 14-29.

Anderson, L.G. and Seijo, J.C. (2010) Bioeconomics of Fisheries Management. Wiley-Blackwell, Singapore.

Anderson, J.L., Anderson, C.M., Chu, J. et al. (2015) The fishery performance indicators: a management tool for triple bottom line outcomes. PLOS ONE 10, e0122809.

Andrew, N., Béne, C., Hall, S.J., Allison, E.H., Heck, S. and Ratner, B.D. (2007) Diagnosis and management of small-scale fisheries in developing countries. Fish and Fisheries 8, 227-240.

Arias, A. (2015) Understanding and managing compliance in the nature conservation context. Journal of Environmental Management 153, 134-143.

Batista, M.I., Baeta, F., Costa, M.J. and Cabral, H.N. (2011) MPA as management tools for small-scale fisheries: the case study of Arrábida Marine Protected Area (Portugal). Ocean $\mathcal{E}$ Coastal Management 54, 137-147.

Baudron, A., Ulrich, C., Nielsen, J.R. and Boje, J. (2010) Comparative evaluation of a mixed-fisheries effortmanagement system based on the Faroe Islands example. Ices Journal of Marine Science 67, 1036-1050.

Béné, C., Macfayden, G. and Allison, E.H. (2006) Increasing the Contribution of Small-Scale Fisheries to Poverty Alleviation and Food Security. FAO Fisheries and Technical Paper No. 481. Food and Agriculture Organization of the United Nations, Rome.

Béné, C., Hersoug, B. and Allison, E.H. (2010) Not by rent alone: analysing the pro-poor functions of smallscale fisheries in developing countries. Development Policy Review 28, 325-358.

Borrell, B. (2013) A big fight over little fish. Nature 493 , 597-598.
Brewer, T.D., Cinner, J.E., Green, A. and Pressey, R.L. (2013) Effects of human population density and proximity to markets on coral reef fishes vulnerable to extinction by fishing. Conservation Biology 27, 443452.

Bruno, J.F. and Selig, E.R. (2007) Regional decline of coral cover in the Indo-Pacific: timing, extent, and subregional comparisons. PLoS ONE 2, e711.

CIESIN (2012) National Aggregates of Geospatial Data Collection: Population, Landscape, and Climate Estimates, Version 3 (PLACE III). NASA Socioeconomic Data and Applications Center (SEDAC), Palisades, NY.

Cinner, J.E., McClanahan, T.R., MacNeil, M.A. et al. (2012) Comanagement of coral reef social-ecological systems. Proceedings of the National Academy of Sciences 109, 5219-5222.

Colloca, F., Cardinale, M., Maynou, F. et al. (2013) Rebuilding Mediterranean fisheries: a new paradigm for ecological sustainability. Fish and Fisheries 14, 89109.

Committee on World Food Security and FAO (2012) Voluntary Guidelines on the Responsible Governance of Tenure of Land, Fisheries, and Forests in the Context of National Food Security. Committee on World Food Security and Food and Agriculture Organization of the United Nations, Rome.

Criddle, K.R. and Strong, J. (2013) Dysfunction by design: consequences of limitations on transferability of catch shares in the Alaska pollock fishery. Marine Policy 40, 91-99.

De'ath, G. and Fabricius, K. (2000) Classification and regression trees: a powerful yet simple technique for ecological data analysis. Ecology 81, 31783192.

Diekert, F.K., Eikeset, A.M. and Stenseth, N.C. (2010) Where could catch shares prevent stock collapse? Marine Policy 34, 710-712.

Essington, T.E., Melnychuk, M.C., Branch, T.A. et al. (2012) Catch shares, fisheries, and ecological stewardship: a comparative analysis of resource responses to a rights-based policy instrument. Conservation Letters $\mathbf{5}$, 186-195.

Fanning, L., Mahon, R. and McConney, P. (2013) Applying the large marine ecosystem (LME) governance framework in the Wider Caribbean Region. Marine Policy 42, 99-110.

FAO (2014) The State of World Fisheries and Aquaculture 2014. Food and Agriculture Organization of the United Nations, Rome.

FAO (2015) Voluntary Guidelines for Securing Sustainable Small-Scale Fisheries in the Context of Food Security and Poverty Eradication. Food and Agriculture Organization of the United Nations, Rome.

FAO Fisheries Department (2003) The ecosystem approach to fisheries. FAO Technical Guidelines for Responsible Fisheries. No. 4, Suppl. 2. 
Finlay, J., McConney, P. and Oxenford, H. (2013) Tenure in the Grenada beach seine fishery. Land Tenure Journal 1, 147-174.

Fujita, R.M., Hopkins, D.D. and Willey, W.R.Z. (1996) Creating incentives to curb overfishing. Forum for Applied Research and Public Policy 11, 29-34.

Geheb, K. and Binns, T. (1997) 'Fishing farmers' or 'farming fishermen'? The quest for household income and nutritional security on the Kenyan shores of Lake Victoria. African Affairs 96, 73-93.

Grafton, R.Q., Arnason, R., Bjorndal, T. et al. (2006) Incentive-based approaches to sustainable fisheries. Canadian Journal of Fisheries and Aquatic Sciences 63 , 699-710.

Grimm, D., Barkhorn, I., Festa, D. et al. (2012) Assessing catch shares' effects evidence from Federal United States and associated British Columbian fisheries. Marine Policy 36, 644-657.

Gutierrez, N.L., Hilborn, R. and Defeo, O. (2011) Leadership, social capital and incentives promote successful fisheries. Nature 470, 386-389.

Harkes, I. and Novaczek, I. (2002) Presence, performance, and institutional resilience of sasi, a traditional management institution in Central Maluku, Indonesia. Ocean \& Coastal Management 45, 237260.

Hersoug, B. and Paulsesn, O. (1996) Monitoring, Control and Surveillance in Fisheries Management. University of Namibia, Windhoek.

Hicks, C.C., Stoeckl, N., Cinner, J.E. and Robinson, J. (2014) Fishery benefits and stakeholder priorities associated with a coral reef fishery and their implications for management. Environmental Science \& Policy 44, $258-270$.

Hilborn, R. and Walters, C.J. (1992) Quantitative Fisheries Stock Assessment: Choice, Dynamics, and Uncertainty. Springer, Norwell.

Hoggarth, D.D., Cowan, V.J., Halls, A.S. et al. (1999) Management Guidelines for Asian Floodplain River Fisheries. Part 1. A Spatial, Hierarchical and Integrated Strategy for Adaptive co-Management. FAO Fisheries and Technical Paper No. 384/1. FAO, Rome, p. 63.

Huang, M.-H. and Ou, C.-H. (2012) A discussion of management disputes arising from the multiple utilization of flying fish resources in Taiwan and suggested countermeasures. Marine Policy 36, 512-519.

Kompas, T. (2005) Fisheries management. Australian Commodities 12, 152-160.

Kompas, T., Che, T.N. and Grafton, R.Q. (2004) Technical efficiency effects of input controls: evidence from Australia's banana prawn fishery. Applied Economics 36, 1631-1641.

Kura, Y., Revenga, C., Hoshino, E. and Mock, G. (2004) Fishing for Answers: Making Sense of the Global Fish Crisis. World Resources Institute, Washington DC, 70 pp.
Lester, S.E. and Halpern, B.S. (2008) Biological responses in marine no-take reserves versus partially protected areas. Marine Ecology-Progress Series 367, 49-56.

Little, L.R., Grafton, R.Q., Kompas, T., Smith, A.D.M., Punt, A.E. and Mapstone, B.D. (2011) Complementarity of no-take marine reserves and individual transferable catch quotas for managing the line fishery of the Great Barrier Reef. Conservation Biology 25, 333-340.

Loh, W.Y. (2011) Classification and regression trees. Wiley Interdisciplinary Reviews-Data Mining and Knowledge Discovery 1, 14-23. [In English].

Maggs, J.Q., Mann, B.Q. and Cowley, P.D. (2013) Contribution of a large no-take zone to the management of vulnerable reef fishes in the South-West Indian Ocean. Fisheries Research 144, 38-47.

Mahon, R., McConney, P. and Roy, R.N. (2008) Governing fisheries as complex adaptive systems. Marine Policy 32, 104-112.

Marchal, P., Andersen, J.L., Aranda, M. et al. (2016) A comparative review of fisheries management experiences in the European Union and in other countries worldwide: Iceland, Australia, and New Zealand. Fish and Fisheries 17, 803-824.

McClanahan, T.R. (1999) Is there a future for coral reef parks in poor tropical countries? Coral Reefs 18, 321325.

Mesnildrey, L., Gascuel, D. and Le Pape, O. (2013) Integrating marine protected areas in fisheries management systems: some criteria for ecological efficiency. Aquatic Living Resources 26, 159-170.

Moreno-Sanchez, R.D.P. and Maldonado, J.H. (2013) Adaptive capacity of fishing communities at marine protected areas: a case study from the Colombian Pacific. Ambio 42, 985-996.

Munday, P.L., Jones, G.P., Pratchett, M.S. and Williams, A.J. (2008) Climate change and the future for coral reef fishes. Fish and Fisheries 9, 261-285.

Nickerson-Tietze, D.J. (2000) Community-based management for sustainable fisheries resources in Phang-nga Bay, Thailand. Coastal Management 28, 65-74.

O'Keefe, C.E., Cadrin, S.X. and Stokesbury, K.D.E. (2013) Evaluating effectiveness of time/area closures, quotas/ caps, and fleet communications to reduce fisheries bycatch. ICES Journal of Marine Science: Journal du Conseil 71, 1286-1297.

Orensanz, J.M. and Seijo, J.C. (2013) Rights-Based Management in Latin American Fisheries. FAO Fisheries and Aquaculture Technical Paper, No. 582.

Ovando, D.A., Deacon, R.T., Lester, S.E. et al. (2013) Conservation incentives and collective choices in cooperative fisheries. Marine Policy 37, 132-140.

Pomeroy, R.S., Cinner, J.E. and Nielsen, J.R. (2011) Conditions for successful co-management: lessons learned in Asia, Africa, the Pacific, and the Wider Caribbean. In: Small-Scale Fisheries Management: Frameworks and 
Approaches for the Developing World. (eds R.S. Pomeroy and N.L. Andrew). CAB International, Oxford, UK.

Poon, S.E., Bonzon, K. and Van Leuvan, T. (2013) Catch Share Design Manual, Volume 2: Cooperative Catch Shares. Environmental Defense Fund, New York.

Ratner, B.D., Oh, E.J.V. and Pomeroy, R.S. (2012) Navigating change: second-generation challenges of smallscale fisheries co-management in the Philippines and Vietnam. Journal of Environmental Management 107, 131-139.

Rice, J. (2006) Impacts of Mobile Bottom Gears on Seafloor Habitats, Species, and Communities: A Review and Synthesis of Selected International Reviews. Research Document 2006/057.

Sciberras, M., Jenkins, S.R., Mant, R., Kaiser, M.J., Hawkins, S.J. and Pullin, A.S. (2015) Evaluating the relative conservation value of fully and partially protected marine areas. Fish and Fisheries 16, 58-77.

Sethi, S.A., Branch, T.A. and Watson, R. (2010) Global fishery development patterns are driven by profit but not trophic level. Proceedings of the National Academy of Sciences 107, 12163-12167.

Stefansson, G. and Rosenberg, A.A. (2005) Combining control measures for more effective management of fisheries under uncertainty: quotas, effort limitation and protected areas. Philosophical Transactions of the Royal Society B-Biological Sciences 360, 133-146.

Sutinen, J.G. and Soboil, M. (2003) The performance of fisheries management systems and the ecosystem challenge. In: Responsible Fisheries in the Marine Ecosystem. (eds M. Sinclair and G. Valdimarsson). CAB International, Oxford, UK.

Teh, L.C.L. and Sumaila, U.R. (2013) Contribution of marine fisheries to worldwide employment. Fish and Fisheries 14, 77-88.

Therneau, T., Atkinson, B. and Ripley, B. (2014) rpart: Recursive Partitioning and Regression Trees. $\mathrm{R}$ package version 4.1-5. Available at: http://cran.r-project.org/ package $=$ rpart (accessed January 2014).

Turner, M.A. (1997) Quota-induced discarding in heterogeneous fisheries. Journal of Environmental Economics and Management 33, 186-195.

United Nations Development Programme (2011) Human Development Report 2011, Sustainability and Equity: A Better Future for All. United Nations Development Programme, New York, NY.

Urquhart, J. and Acott, T. (2014) A sense of place in cultural ecosystem services: the case of Cornish fishing communities. Society \& Natural Resources 27, 3-19. [In English].

Villasante, S. and Sumaila, U.R. (2010) Estimating the effects of technological efficiency on the European fishing fleet. Marine Policy 34, 720-722.
Walmsley, S.F. and White, A.T. (2003) Influence of social, management and enforcement factors on the long-term ecological effects of marine sanctuaries. Environmental Conservation 30, 388-407.

Wamukota, A.W., Cinner, J.E. and McClanahan, T.R. (2012) Co-management of coral reef fisheries: a critical evaluation of the literature. Marine Policy 36, 481488.

Weeratunge, N., Béné, C., Siriwardane, R. et al. (2013) Small-scale fisheries through the wellbeing lens. Fish and Fisheries 15, 255-279.

Wejnert, B. (2002) Integrating models of diffusion of innovations: a conceptual framework. Annual Review of Sociology 28, 297-326.

Wielgus, J., Poon, S., Carballal del Rio, E., Munoz, D., Whittle, D. and Fujita, R. (2014) Fishery cooperatives in Cuba: potential benefits, legal feasibility, and governance pre-conditions. Marine Policy 45, 128-137.

Yamazaki, S., Grafton, Q.R., Kompas, T. and Jennings, S. (2012) Biomass management targets and the conservation and economic benefits of marine reserves. Fish and Fisheries 15, 196-208.

\section{Supporting Information}

Additional Supporting Information may be found in the online version of this article:

Figure S1. Classification trees by management tool with region.

Table S1. Search terms from English-source peer-reviewed literature used to identify studies assessing effectiveness of different management tools.

Table S2. Number of studies per region and number of times a management tool was recorded in the literature by region.

Table S3. Coefficients, standard errors, and $P$ values for the limited entry GLM.

Table S4. Coefficients, standard errors, and $P$ values for the gear restriction GLM.

Table S5. Coefficients, standard errors, and $P$ values for the quotas GLM.

Table S6. Coefficients, standard errors, and $P$ values for the MPA GLM.

Table S7. Coefficients, standard errors, and $P$ values for the time-area closure GLM.

Table S8. Coefficients, standard errors, and $P$ values for the size limits GLM. 